\title{
dCIP4 (Drosophila Cdc42-Interacting Protein 4) Restrains Synaptic Growth by Inhibiting the Secretion of the Retrograde Glass Bottom Boat Signal
}

\author{
Minyeop Nahm, ${ }^{1,3 \star}$ Sungdae Kim, ${ }^{2,3 \star}$ Sang Kyoo Paik, ${ }^{4}$ Mihye Lee, ${ }^{3}$ Seongsoo Lee, ${ }^{3}$ Zang Hee Lee, ${ }^{3}$ Jaesang Kim, ${ }^{5}$ \\ Daekee Lee, ${ }^{5}$ Yong Chul Bae, ${ }^{4}$ and Seungbok Lee ${ }^{1,2,3}$ \\ ${ }^{1}$ Interdisciplinary Graduate Program in Brain Science, ${ }^{2}$ Interdisciplinary Graduate Program in Genetic Engineering, and ${ }^{3}$ Department of Cell and \\ Developmental Biology, Dental Research Institute, Seoul National University, Seoul 110-740, Republic of Korea, ${ }^{4}$ Department of Oral Anatomy and \\ Neurobiology, School of Dentistry, Kyungpook National University, Daegu 700-412, Republic of Korea, and ${ }^{5}$ Division of Life and Pharmaceutical Sciences, \\ Ewha Womans University, Seoul 120-750, Republic of Korea
}

The bone morphogenetic protein (BMP) ligand Glass bottom boat (Gbb) acts as a retrograde growth signal at the Drosophila neuromuscular junction (NMJ). Endocytic regulation of presynaptic BMP receptors has been proposed to attenuate retrograde BMP signaling. However, it remains unknown whether the Gbb signal is also regulated by postsynaptic mechanisms. Here, we provide evidence that Drosophila Cdc42-interacting protein 4 (dCIP4) functions postsynaptically to inhibit synaptic growth. dCIP4 is localized postsynaptically at NMJs. dcip4 mutations lead to synaptic overgrowth and increased presynaptic phosphorylated mothers against decapentaplegic (Mad) levels, and these defects are rescued by muscle-specific expression of dCIP4. Biochemical and genetic analyses demonstrate that dCIP4 acts downstream of Cdc42 to activate the postsynaptic Wsp-Arp2/3 pathway. We also show that BMP signaling is necessary for synaptic overgrowth in larvae lacking postsynaptic dcip4 or wsp. Finally, dCIP4 and Wsp inhibit Gbb secretion. Thus, we propose that dCIP4 restrains synaptic growth by inhibiting postsynaptic Gbb secretion through the Wsp-Arp2/3 pathway.

\section{Introduction}

Synapses are specialized intercellular junctions that allow efficient communication between neurons and their postsynaptic targets. It is now well established that retrograde signals derived from postsynaptic cells play critical roles in synaptic development and plasticity (Tao and Poo, 2001; Marques and Zhang, 2006; Regehr et al., 2009). At the Drosophila neuromuscular junction $(\mathrm{NMJ})$, the bone morphogenetic protein (BMP) homolog Glass bottom boat (Gbb) acts as a muscle-derived retrograde signal that promotes synaptic growth and neurotransmitter release (McCabe et al., 2003). This signal is conveyed into presynaptic neurons through a receptor complex consisting of the type-II receptor Wishful thinking (Wit) and either of two type-I receptors, Thickveins (Tkv) and Saxophone (Sax) (Aberle et al., 2002; Marques et al., 2002; Rawson et al., 2003). Receptor activation results in the phosphorylation of mothers against decapentaple-

Received Jan. 15, 2010; revised April 1, 2010; accepted April 29, 2010.

This work was supported by grants from the Brain Research Center of the 21st Century Frontier (2009K001278) the Research Program for New Drug Target Discovery (M10748000283-07N4800-28310), the Korea Research Foundation (KRF-2006-312-C00361), the National Research Foundation (NRF-2006-511-C00100) (M.N.), and the Seoul Science Fellowship (S.K.). We are very grateful to Giuseppa Pennetta and Frank Yu for valuable comments on this manuscript. We would like to thank Young-Ho Koh for the BG57-GAL4 line, Sven Bogdan for the anti-Wsp antibody, Aaron DiAntonio for the anti-GluRIIB antibody, Haig Keshishian for the MHC-GS-GAL4 line, Peter ten Dijke for the anti-P-Mad antibody, Akira Chiba for the UAS-Myc-cdc42V12 line, and Eyal Schejter for the wsp ${ }^{7}$ and Df(3R)3450 lines.

${ }^{*}$ M.N. and S.K. contributed equally to this work.

Correspondence should be addressed to Seungbok Lee at the above address. E-mail: seunglee@snu.ac.kr.

DOI:10.1523/JNEUROSCI.0256-10.2010

Copyright $\odot 2010$ the authors $\quad 0270-6474 / 10 / 308138-13 \$ 15.00 / 0$ gic (Mad). Phosphorylated Mad (P-Mad) enters the nucleus to regulate transcription of target genes (Keshishian and Kim, 2004).

A growing body of evidence suggests that endocytosis is a key mechanism for the regulation of retrograde BMP signaling during synaptic growth. Mutations in various endocytic genes, including dap160/intersectin, spin, and spichthyin (spict), cause synaptic overgrowth (Sweeney and Davis, 2002; Marie et al., 2004; Wang et al., 2007), and this phenotype is suppressed by additional mutations that disrupt BMP signaling (Sweeney and Davis, 2002; Wang et al., 2007; O'Connor-Giles et al., 2008), suggesting that endocytosis contribute negatively to retrograde BMP signaling. However, it is not known whether retrograde BMP signaling is also regulated at the level of Gbb secretion.

The Drosophila ortholog of Wasp (Wsp) has been implicated in endocytic regulation of retrograde BMP signaling. Wsp is required for restraining synaptic growth and binds to the presynaptic protein Nervous wreck (Nwk) (Coyle et al., 2004). Nwk can interact with $\mathrm{Tkv}$ and dynamin and Dap160/intersectin (O'Connor-Giles et al., 2008), suggesting that Wsp function is critical for endocytic regulation of BMP receptors. However, most Wsp at the NMJ is present postsynaptically (Coyle et al., 2004), suggesting that Wsp can regulate synaptic growth independently of Nwk.

Drosophila Cdc42-interacting protein 4 (dCIP4) is a multimodular adaptor protein that contains an N-terminal Fes/CIP4 homology-BAR (F-BAR) domain followed by a central Cdc42interacting protein kinase $\mathrm{C}$-related kinase homology region 1 
(HR1) domain and a C-terminal Src homology region 3 (SH3) domain, which interacts with dynamin and Wsp (Leibfried et al., 2008). The F-BAR domain of dCIP4 has a potent activity to induce plasma membrane invagination (Fricke et al., 2009). In epithelial cells, dCIP4 has been shown to regulate dynamindependent endocytosis of E-cadherin at adherens junctions by acting downstream of Cdc42 to activate the Wsp-Arp2/3mediated actin polymerization (Georgiou et al., 2008; Leibfried et al., 2008; Fricke et al., 2009).

Here, we examined the role of dCIP4 at the NMJ. Our experiments demonstrate that $\mathrm{dCIP} 4$ is required postsynaptically to restrain synaptic growth. We also show that dCIP4 acts positively in the Cdc42-Wsp pathway to inhibit postsynaptic Gbb secretion. These results reveal a novel role for dCIP4 in synaptic growth regulation and demonstrate that retrograde BMP signaling is regulated at the level of Gbb secretion from postsynaptic cells.

\section{Materials and Methods}

Fly stocks. The wild-type strain was $w^{1118}$ unless otherwise noted. An enhancer-promoter (EP) insertion in the dcip4 locus (G14250) was purchased from GenExel. The $d c i p 4$ allele $d c i p 4^{1}$ was generated via imprecise excision of G14250. Transgenic lines carrying UAS-HA-dcip4, UAS-HAdcip4-L145D, UAS-HA-dcip4-L436S, UAS-HA-dcip4-W603K, or UAS$g b b$-GFP were generated in the $w^{1118}$ background by standard procedures (Robertson et al., 1988). $w s p^{1}$ and $D f(3 R) 3450$ (a deficiency in the $w s p$ locus) flies were kindly provided by Eyal Schejter (Weizmann Institute of Science, Rehovot, Israel), UAS-Myc-cdc42V12 (Kim et al., 2003) by Akira Chiba (University of Miami, Coral Gables, FL), UAS-cdc42V12 and UAScdc42N17 (Luo et al., 1994) by Peter Kolodziej (Vanderbilt University, Nashville, TN), shits by Anne Schmidt (University of Heidelberg, Heidelberg, Germany), and $D f(3 L) E D 4342$ (a deficiency in the dcip4 locus), $c d c 42^{2}, w i t^{A 12}$, wit ${ }^{B 11}, \operatorname{arp}^{E P 3640}$, and UAS-shi ${ }^{K 44 A}$ (Moline et al., 1999) by the Bloomington Drosophila Stock Center. UAS-dcip $4^{R N A i}(+278$ to +778 ) was obtained from the National Institute of Genetics Stock Center Mishima, Japan. Other RNA interference (RNAi) lines were obtained from Vienna Drosophila RNAi Center: $U A S-c d c 42^{R N A i}(+294$ to +642$)$, $U A S-w s p^{R N A i}(+434$ to +773$), U A S-\operatorname{arp} 2^{R N A i}(+633$ to +968$)$, and $U A S-\operatorname{arp} 3^{R N A i}(+544$ to +887$)$. The following GAL4 lines were used in this study: BG57-GAL4 (Budnik et al., 1996), MHC-GeneSwitchGAL4 (MHC-GS-GAL4) (Osterwalder et al., 2001), and C155-GAL4 (Lin and Goodman, 1994).

Molecular biology. For glutathione S-transferase (GST) pull-down assays, a cDNA fragment spanning the entire dcip4 open reading frame (ORF) was amplified by PCR from the expressed sequence tag (EST) clone RE39037 and subcloned into the $p$ GEX6P1 vector (GE Healthcare). L145D, L436S, and W603K mutations were introduced into the pGEX6P1-dcip4 construct using the QuikChange Multi kit (Stratagene). A DNA fragment encoding the Wsp proline-rich domain (amino acids 301-378) was also amplified by PCR from EST clone RE12101 and introduced into $p$ GEX6P1. For mammalian expression of Myc-tagged wildtype and mutant dCIP4 proteins, the same cDNA fragments were inserted into the $p C M V$-Tag $3 B$ vector (Stratagene). For mammalian expression of green fluorescent protein (GFP)-tagged Wsp and Gbb, the entire coding sequences of $w s p$ and $g b b$ were amplified by PCR from EST clones RE12101 and GH12092, respectively, and then subcloned into the pEGFP-N2 vector (Clontech). For mammalian expression of Myc-tagged Cdc42V12 and Cdc42N17, their entire coding sequences were PCR amplified from genomic DNAs of transgenic flies carrying $U A S-c d c 42 V 12$ and $U A S-c d c 42 N 17$, respectively, and then subcloned into $p C M V-T a g 3 B$. For mammalian expression of hemagglutinin (HA)-tagged Cdc42V12, the insert of $p C M V-T a g 3 B-c d c 42$ V 12 was subcloned into the $p c D N A-H A$ vector (Invitrogen). For transgenic rescue experiments, the dcip4, dcip4L145D, dcip4-L436S, and dcip4-W603K cDNA inserts in pGEX6P1 were introduced into $P U A S T-H A$, a derivative of the $P U A S T$ vector (Brand and Perrimon, 1993). The following primers were used to characterize the excision line dcip $4^{1}$ at the molecular level: $5^{\prime}$ -
ACACTTGGTAAGCTTTTTCAGAGC-3' and 5'-GTTCGGGATCCTCCTTGAGCTGCT-3'.

To evaluate the effects of the $d c i p 4^{1}$ mutation on the expression levels of dcip4, mRpS6, and rp49 (control) mRNAs, total RNA was isolated from larval extracts using TRIzol (Invitrogen). Reverse transcription (RT) was performed with $1 \mu \mathrm{g}$ RNA, an oligo-dT primer, and the SuperScript II reverse transcription kit (Invitrogen). The resulting cDNA was analyzed by PCR using the following primers: dcip4, 5'-CGAAGAAAGAAACTGCAG-3' and 5'-GGGACTTGCCGAACCGTT-3'; mRpS6, 5'-AATTGCCTCGCCCCGAACTGA-3' and $5^{\prime}$-TAGCATACATAATTA ACGCGTTT-3'; rp49, 5' -CACCAGTCGGATCGATATGC-3' and 5'-CACGTTGTGCACCAGGAACT-3'.

Cell culture and transient transfection. Drosophila Schneider S2R + cells were maintained at $25^{\circ} \mathrm{C}$ in Schneider's medium (Invitrogen) supplemented with $10 \%$ heat-inactivated fetal bovine serum (FBS). Human embryonic kidney 293T (HEK293T) cells were grown in DMEM containing 10\% heat-inactivated FBS. S2R + and HEK293T cells were transfected in six-well plates using Cellfectin (Invitrogen) and Lipofectamine 2000 (Invitrogen), respectively, according to the manufacturer's instructions.

Generation of dCIP4 antibody. To generate a polyclonal antibody against dCIP4, a recombinant protein containing a fragment of dCIP4 (amino acids 201-567) fused with GST at the $\mathrm{N}$ terminus was expressed in Escherichia coli BL21 (Stratagene). The fusion protein was purified with glutathione-Sepharose $4 \mathrm{~B}$ and digested with PreScission protease (GE Healthcare). The cleaved dCIP4 fragment was further purified by SDS-PAGE for the immunization of rats. The resulting sera were affinity purified using the antigen-cross-linked to $\mathrm{CNBr}$-activated Sepharose 4 Fast Flow beads (GE Healthcare).

Western blot analysis. Larval body-wall muscle preparations were homogenized in SDS sample buffer and then boiled for $5 \mathrm{~min}$. S2R + cells were homogenized in $50 \mathrm{~mm}$ Tris- $\mathrm{HCl}, \mathrm{pH} 7.5,150 \mathrm{~mm} \mathrm{NaCl}, 1 \%$ Triton $\mathrm{X}-100$, and $1 \times$ protease inhibitor cocktail (Roche) and mixed with SDS sample buffer. After boiling for $5 \mathrm{~min}$, the homogenates were subjected to SDS-PAGE and transferred to nitrocellulose membranes (Whatman). Protein bands were visualized with an ECL detection system (Pierce). The following antibodies were used in this study: rat anti-dCIP4 (1: 1000), guinea pig anti-Wsp (a kind gift from Sven Bogdan, Universität Münster, Münster, Germany; 1:1000), rabbit anti-dynamin (a kind gift from Mani Ramaswami, Trinity College Dublin, Dublin, Ireland; 1:1000), rabbit anti-Actin (Sigma; 1:1000), rabbit anti-Myc (Cell Signaling Technology; 1:1000), mouse anti-GFP (Roche; 1:1000), and HRPconjugated secondary antibodies (Jackson ImmunoResearch; 1:5000).

GST pull-down assays. GST fusion proteins of dCIP4, dCIP4-L436S, dCIP4-W603K, Wsp-PRD, and GST alone were produced in E. coli and purified using glutathione-Sepharose 4B (GE Healthcare). HEK293T cells transiently expressing Myc-Cdc42V12, HA-Cdc42V12, MycCdc42N17 or Wsp-GFP were lysed in $20 \mathrm{~mm}$ Tris-HCl, pH 8.0, $150 \mathrm{~mm}$ $\mathrm{NaCl}, 1 \mathrm{~mm}$ EDTA, and $0.5 \% \mathrm{NP}-40$, and then centrifuged at $12,000 \times \mathrm{g}$ for $15 \mathrm{~min}$ at $4^{\circ} \mathrm{C}$. The cell lysates $(500 \mu \mathrm{l})$ were incubated with $10 \mu \mathrm{g}$ of GST or GST fusion proteins immobilized on glutathione-Sepharose beads for $4 \mathrm{~h}$ at $4^{\circ} \mathrm{C}$. Beads were washed three times with lysis buffer and boiled in SDS sample buffer. The eluates were subjected to Western blot analysis using anti-Myc, anti-HA, or anti-GFP.

Immunohistochemistry. Whole-mount staining of embryos was performed as described previously (Lee et al., 2000). Brains were dissected out from wandering third instar larvae in PBS, fixed in $0.1 \mathrm{M}$ PIPES, $\mathrm{pH}$ 6.9, $1 \%$ formaldehyde, $1 \mathrm{~mm}$ EGTA, $1 \%$ Triton X-100, and $2 \mathrm{~mm} \mathrm{MgSO}_{4}$ for $30 \mathrm{~min}$ at $4^{\circ} \mathrm{C}$, and washed with wash buffer ( $50 \mathrm{~mm}$ Tris- $\mathrm{HCl}, \mathrm{pH} 6.8$, $150 \mathrm{~mm} \mathrm{NaCl}, 0.5 \% \mathrm{NP}-40,1 \mathrm{mg} / \mathrm{ml} \mathrm{BSA}$ ). Larval body wall muscles were dissected from wandering third instar larvae in $\mathrm{Ca}^{2+}$-free HL3 saline (Stewart et al., 1994) and fixed in 4\% formaldehyde in PBS for $30 \mathrm{~min}$. Fixed brain and muscle samples were washed with PBT (PBS, 0.1\% Triton X-100), blocked with 5\% BSA/PBT for $1 \mathrm{~h}$, and incubated with primary antibodies overnight at $4^{\circ} \mathrm{C}$. Samples were incubated with secondary antibodies for $1 \mathrm{~h}$ at room temperature. The following primary antibodies were used: rat anti-dCIP4 (1:100), guinea pig anti-Wsp (1:100), rabbit anti-P-Mad (1:100) (Persson et al., 1998), goat anti-HRP conjugated with FITC (Jackson ImmunoResearch; 1:200), mouse anti- 
Dlg (Developmental Studies Hybridoma Bank; 1:500), mouse antiGluRIIA (Developmental Studies Hybridoma Bank; 1:10), rabbit antiGluRIIB (1:2500), and mouse anti-synaptotagmin (anti-Syt; Developmental Studies Hybridoma Bank; 1:5). FITC-, cyanine 3 (Сy3)-, and Cy5conjugated secondary antibodies (Jackson ImmunoResearch) were used at a dilution of 1:200. Muscle actin was visualized using rhodamine-conjugated phalloidin (Invitrogen; 1:150).

Morphological quantification. Fluorescence images of larval NMJs were acquired using an Olympus FV300 laser-scanning confocal microscope. To compare different genotypes, samples were processed simultaneously and imaged under identical confocal settings. For postsynaptic F-actin quantification, NMJs on muscles 12 and 13 were analyzed to minimize interference from $\mathrm{F}$-actin within underlying myofibrils as described previously (Ramachandran et al., 2009). All other quantifications were performed at muscles 6 and 7 in abdominal segment 2. For quantification of NMJ morphology, a complete z-series stack collected at intervals of $1 \mu \mathrm{m}$ was projected using the maximum intensity method. Total bouton numbers, satellite bouton numbers, and NMJ lengths were measured after anti-HRP staining. NMJ length was determined by summing the length of synaptic branches. A synaptic branch was defined as an arborization with two or more type I boutons. Satellite boutons were defined as single boutons that were not included in a chain of boutons, as described previously (Estes et al., 2003). Muscle surface area was visualized by saturating HRP signal and measured using Olympus FLOUVIEW (version 5.0) image analysis software. Bouton numbers and NMJ lengths were normalized to the muscle surface area. For P-Mad and postsynaptic F-actin quantification, the fluorescence intensities of P-Mad and phalloidin were measured using the FLOUVIEW software and then normalized to HRP intensity.

The numbers of samples analyzed are indicated inside the bars in Figures 2 and $5-8$. The data are presented as mean \pm SEM. Statistical significance was determined by one-way ANOVA using SPSS software (version 17.0). Significant effects were analyzed further by post hoc pairwise comparisons of means using Turkey-Kramer or Fisher's least significant difference test (statistically significant for $p<0.05$ ).

RNA interference and Gbb secretion assays. For RNAi experiments in $\mathrm{S} 2 \mathrm{R}+$ cells, double-stranded RNAs (dsRNAs) were generated by in vitro transcription of DNA templates containing T7 promoter sequences at both ends as described previously (Lee et al., 2007). DNA templates were amplified by PCR using primers containing the T7 promoter sequence upstream of the following: dcip4, $5^{\prime}$-AATTCCCTGACTGCGGATGG-3' and 5'-GAATGTCTAGAGGTTCGGTG-3'; wsp, 5'-GATGGTCATGTGGGACTAAA-3' and 5'-GATGGATGACGGGTTGGCAC-3'; arp3, 5'-CGAGGGCTATGTGATCGGCT- ${ }^{\prime}$ and $5^{\prime}$-GATCCGGAGACGTGTGTCCA-3'; shi, 5'-GGAGTTACCGAATATGGC-3' and 5'-ATCTATTCACCACGCCAA-3'.

To determine the effect of depleting dCIP4, Wsp, Arp3, or dynamin on Gbb secretion, S2R + cells transiently expressing Gbb-GFP were treated with $5 \mu \mathrm{g} / \mathrm{ml}$ of dsRNA targeting dcip4, wsp, arp3, or shi for $5 \mathrm{~h}$ in serum-free medium. Cells were maintained in serum-containing medium for $4 \mathrm{~d}$ (dcip4, wsp, and $\operatorname{arp} 3)$ or $7 \mathrm{~d}(s h i)$ to allow for protein depletion. To determine the effect of dCIP4 or Wsp overexpression on Gbb secretion, S2R + cells were transiently transfected with both GbbGFP and dCIP4 or Wsp constructs and maintained in serum-containing medium for $2 \mathrm{~d}$. All transfected cells were fed with fresh serum-free medium and grown for an additional $12 \mathrm{~h}$. The medium was collected for Western blot analysis using mouse anti-GFP. Cells were harvested in 50 mм Tris, $\mathrm{pH} 7.5,150 \mathrm{~mm} \mathrm{NaCl}, 1 \%$ Triton X-100, and protease inhibitors (Roche) at $4^{\circ} \mathrm{C}$, and then centrifuged at $12,000 \times g$ for $15 \mathrm{~min}$. Supernatants were precleared with protein A/G PLUS-agarose (Santa Cruz Biotechnology) for $1 \mathrm{~h}$ at $4^{\circ} \mathrm{C}$. The samples were incubated with rabbit anti-GFP (Abcam; 1:100) for $4 \mathrm{~h}$ at $4^{\circ} \mathrm{C}$ and then incubated with protein $\mathrm{A} / \mathrm{G}$ PLUS-agarose for $2 \mathrm{~h}$ at $4^{\circ} \mathrm{C}$. Beads were washed three times with PBS and boiled in SDS sample buffer. The eluates were subjected to Western blotting using mouse anti-GFP. To determine the efficacy of dsRNAs and to ensure equal amounts of staring material, cell lysates were analyzed by Western blotting using anti-dCIP4, anti-Wsp, antidynamin, or anti- $\beta$-actin, or by RT-PCR analysis using arp3-specific primers.

\section{Results}

\section{Isolation of $d c i p 4$ mutants}

When stained with an antibody against the axonal membrane marker HRP (Jan and Jan, 1982), the Drosophila NMJ at the crawling third instar stage displays stereotyped, segment-specific numbers of branches and boutons. Using this histochemical approach, we screened an EP collection (Lee et al., 2005) for mutations that affect larval NMJ morphology. We thereby isolated a P-element insertion (G14250) localized in the 5' untranslated region of the Drosophila cip4 gene (dcip4) (Fig. 1A). This P-element mutant was homozygous viable and had more extensive NMJs than those of a wild-type control $\left(w^{1118}\right)$ (data not shown). The predicted dCIP4 protein harbors an N-terminal F-BAR domain (amino acids 31-312) followed by an HR1 domain (amino acids 375-455) and a C-terminal SH3 domain (amino acids 568-629), identical to the human CIP4 protein (Fig. 1A). Overall, dCIP4 displays 35\% identity and 64\% similarity to human CIP4 at the amino acid level.

To address the role of dCIP4 at the NMJ, we generated an additional dcip4 mutant $\left(d c i p 4^{1}\right)$ by imprecise excision of G14250. The $d c i p 4^{1}$ allele had a deletion within the dcip4 locus, which removes a part of the first exon including the initiation codon, the first intron, and the second exon of the dcip 4 gene (Fig. 1A). dcip 4 mRNA was not expressed in homozygous $d c i p 4^{1}$ larvae (data not shown) or in larvae of $d c i p 4^{1}$ heterozygous with the deficiency $D f(3 L) E D 4342$ deleting the dcip4 locus (Fig. $1 B$ ), suggesting that $d c i p 4^{1}$ is null for the dcip 4 gene. In contrast, mRNA of the $m R p S 6$ gene that is located adjacent to the dcip4 gene was normally expressed in the same larvae (Fig. 1B). The dcip $4^{1} /$ $d c i p 4^{1}$ and $d \operatorname{cip} 4^{1} / D f(3 L) E D 4342$ animals were semilethal at the pupal stage with an incidence of lethality of $\sim 17 \%$. Ubiquitous expression of UAS-HA-dcip4 under the control of da-GAL4 (Wodarz et al., 1995) led to a nearly complete rescue of this lethality.

\section{dCIP4 is required postsynaptically for normal synaptic growth}

Initial examination of third instar $d c i p 4^{1} / D f(3 L) E D 4342$ larvae revealed that the phenotype of synaptic overgrowth is apparent at all type I NMJs, including NMJ 6/7 and NMJ 4 (Fig. $2 A, B$ ). To quantify the dcip 4 phenotype, we counted overall bouton number, satellite bouton number, and NMJ length at NMJ 6/7 in abdominal segment 2 (Fig. 2; supplemental Table 1, available at www.jneurosci.org as supplemental material). Compared with the wild-type control $\left(w^{1118}\right)$, bouton number normalized to muscle surface area was increased by $\sim 32 \%(p<0.001)$ in dcip $4^{1} / D f(3 L) E D 4342$ mutant larvae (Fig. $2 G$ ). In addition, satellite bouton number and NMJ length normalized to muscle surface area were increased by $67 \%(p<0.001)$ and $24 \%(p<$ 0.001 ), respectively (Fig. $2 H, I$ ). Larvae homozygous for $d_{c i p} 4^{1}$ displayed NMJ growth defects that were virtually identical to those of $d c i p 4^{1} / D f(3 L) E D 4342$ larvae (data not shown).

To determine whether dCIP4 is required in the neuron or the muscle for normal synaptic growth, we expressed an HA epitopetagged dcip 4 transgene (UAS-HA-dcip4 ) in dcip $4^{1} / D f(3 L) E D 4342$ animals under the control of tissue-specific GAL4 drivers. Expression of UAS-HA-dcip4 using the muscle-specific BG57-GAL4 driver fully rescued the synaptic overgrowth phenotype of $d c i p 4$ mutants (Fig. 2C, $G-I$, dCIP4 rescue-post). However, expression of the same transgene using the neuronal C155-GAL4 driver had no effect (Fig. 2G-I, dCIP4 rescue-pre). Thus, we concluded that $\mathrm{dCIP} 4$ may function primarily in postsynaptic muscles to regulate synaptic growth. 
A
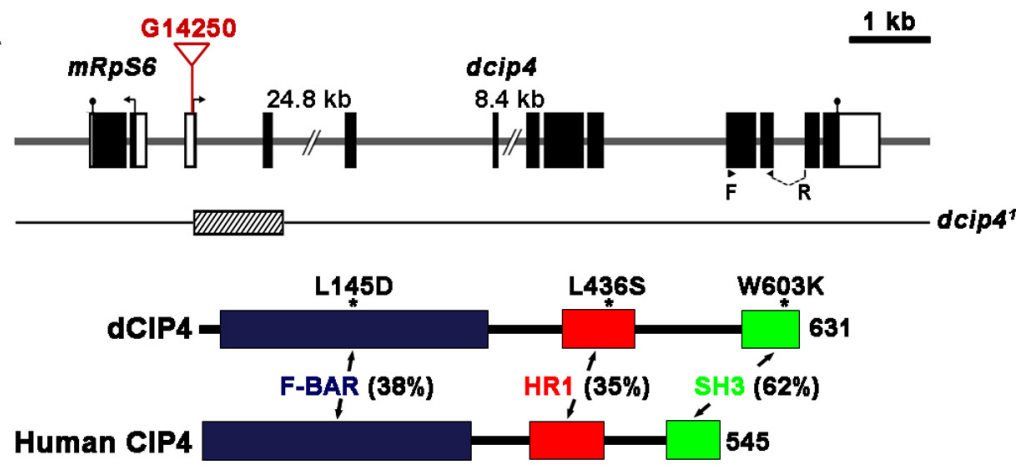

B

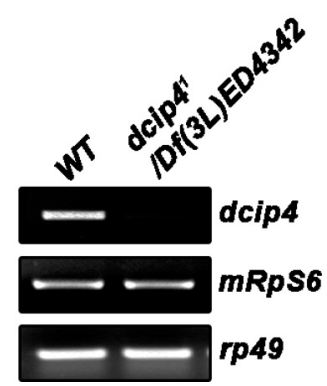

C

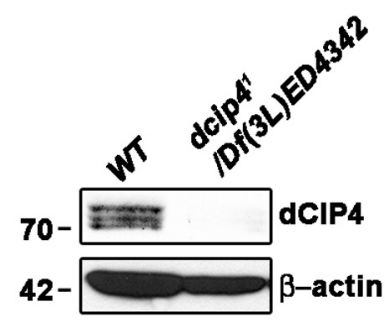

Figure 1. Molecular characterization of a dcip4-null mutant. A, Genomic organization of the dcip4 locus at 64B1-2 on chromosome III and mapping of a dcip4-null mutant. The exon-intron structures of dcip4 and its adjacent gene $m R p S 6$ are shown at the top, along with their translation start (arrows) and stop (closed circle) sites. Introns are indicated by horizontal lines, and exons by boxes. White and black boxes represent untranslated and coding regions, respectively. The insertion site of the P-element line G14250 is indicated as the inverted triangle in the $5^{\prime}$ untranslated region of dcip4 ( $\sim 75$ bp upstream of the initiator ATG). F and R indicate the locations of primers used for RT-PCR analysis of $d c i p 4$ mRNA. The extent of the deletion in $d c i p 4^{1}$, which was generated by imprecise excision of G14250, is marked by the hatched box. Shown below are domain structures of dCIP4 and human CIP4, with the percentage of identity between their corresponding domains. The single asterisks denote substitution mutations of dCIP4 used in this study. F-BAR is shown in blue, HR1 in red, and SH3 in green. B, RT-PCR analysis of dcip4, mRpS6, and rp49 in third instar wild-type (WT) larvae $\left(w^{1118}\right)$ and dcip4 $4^{1}$ IDf(3L)ED4342 mutant larvae. C, Western blot of total extracts of third instar wild-type and dcip4 $4^{1} / D f(3 L) E D 4342$ larvae probed with anti-dCIP4 and reprobed with anti- $\beta$-actin. Numbers on the left are molecular masses in kilodaltons.

dCIP4 is highly enriched in the central nervous system and localizes to the postsynaptic side of NMJs

To characterize the dCIP4 protein at the NMJ, we generated an antibody against its N-terminal region (amino acids 201-567). In Western blot analysis of wild-type $\left(w^{1118}\right)$ third instar larval extracts, our anti-dCIP4 antibody detected three protein bands ranging from 70 to $74 \mathrm{kDa}$, all of which were absent from extracts from $d c i p 4^{1} / D f(3 L) E D 4342$ mutant larvae (Fig. 1C). Because the predicted weight of dCIP4 is $\sim 70 \mathrm{kDa}$, the two additional bands may represent posttranslational modifications of the protein. Consistent with this notion, HA-tagged full-length dCIP4 transiently expressed in S2 cells was also detected as three bands in Western blot analysis using an anti-HA antibody (data not shown).

Immunohistochemical analysis using anti-dCIP4 revealed that dCIP4 is maternally contributed and, in embryos at cellular blastoderm stages, is localized to the cell borders (Fig. 3A1--A3, stage 5). From embryonic stages 12 and 13, dCIP4 immunoreactivity was most prominent in the developing CNS (Fig. 3B). In later stage embryos (stages 16 and 17), dCIP4 was detected in the CNS, the salivary gland, and the surface epithelium (Fig. 3C; supplemental Fig. 1, available at www.jneurosci.org as supplemental material). Double staining with antibodies to presynaptic and postsynaptic markers (Syt and Dlg, respectively) revealed that dCIP4 is present in synaptic regions of the embryonic ventral nerve cord (VNC) (supplemental Fig. 1, available at www.jneurosci. org as supplemental material). We did not detect any dCIP4 immunoreactivity in dcip $4^{1} / D f(3 L) E D 4342$ mutant embryos (Fig. 3D), confirming the specificity of our anti-dCIP4 antibody.

We also analyzed the synaptic localization of dCIP4 in the larval nervous system. Double staining of third instar brains using anti-dCIP4 together with anti-Syt or anti-Dlg revealed that $\mathrm{dCIP} 4$ is present in several synaptic neuropil regions of the brain lobes and VNC (Fig. 3E1--F3). In addition to its CNS expression, dCIP4 was detected at glutamatergic type Ib and Is boutons of the larval body-wall muscles, but it was absent from type II/III boutons and the cytoplasm of muscle cells (Fig. 3G1--G3). Comparison of dCIP4 immunoreactivity with that of HRP revealed that dCIP4 localization at the larval NMJs is mostly postsynaptic (Fig. 3G3, inset). dCIP4 was undetectable at the NMJs of dcip $4^{1} / D f(3 L) E D 4342$ mutant larvae (Fig. $3 H 1--H 3)$, demonstrating the specificity of dCIP4 staining. The postsynaptic localization of dCIP4 was confirmed by its strong overlap with Dlg (Fig. 3I1--I3), a well-established marker of the subsynaptic reticulum (Lahey et al., 1994). Interestingly, dCIP4 immunoreactivity was extended slightly beyond the Dlg-positive region into the muscle and often appeared in a punctate pattern (Fig. 3I1--I3, insets). Thus, we concluded that dCIP4 is a novel postsynaptic component in Drosophila.

The Cdc42- and Wsp-binding activities of dCIP4, but not its membrane-tubulation activity, are essential for normal synaptic growth

dCIP4 can induce membrane tubulation via its F-BAR domain (Fricke et al., 2009). In addition, the HR1 and SH3 domains of dCIP4 have been shown to interact, respectively, with the activated form of Cdc42 and its downstream effector Wsp (Leibfried et al., 2008). In an initial attempt to investigate the mechanism underlying dCIP4-mediated regulation of synaptic growth, we extended these previous findings. First, we demonstrated that the point mutation L145D in dCIP4 completely abolished its ability to induce membrane tubulation in cultured cells (supplemental Fig. $2 A, B$, available at www.jneurosci.org as supplemental material). An analogous mutation (F117D) in mammalian CIP4 was also shown to abolish the induction of membrane tubulation (Frost et al., 2008). Second, after confirming the interactions between dCIP 4 and the activated form of Cdc42 and Wsp by GST pull-down assays, we generated dCIP4 mutants defective in either Cdc42 or Wsp binding. The point mutation I398S in the CIP4 HR1 domain abolishes its binding to active Cdc42 (Tian et al., 2000), and the W518K mutation in the SH3 domain of Toca-1, a close relative of CIP4, abrogates its interaction with N-Wasp (Ho et al., 2004). We found that the L436S mutation in dCIP4 analogous to the CIP4-I398S mutation completely abolished its interaction with an active mutant form of Cdc42 (Cdc42V12), whereas dCIP4-Wsp interaction was not affected by the same mutation in dCIP4 (supplemental Fig. $2 C$, available at www. 
jneurosci.org as supplemental material). In contrast, the W603K mutation in $\mathrm{dCIP} 4$ analogous to the Toca-1-W518K mutation impaired the dCIP4-Wsp interaction but not the dCIP4-Cdc42 interaction (supplemental Fig. 2C, available at www. jneurosci.org as supplemental material). Finally, we demonstrated that dCIP4 is able to interact with activated $\mathrm{Cdc} 42$ and Wsp simultaneously in vitro (supplemental Fig. $2 D$, available at www.jneurosci.org as supplemental material), suggesting that $\mathrm{dCIP} 4$ can act as an adaptor protein in the Cdc42-Wsp pathway.

To determine which domains of dCIP4 are essential for the regulation of synaptic growth, we put UAS transgenes for HAdCIP4-L145D, HA-dCIP4-L436S, and HA-dCIP4-W603K into the dcip $4^{1}$ / $D f(3 L) E D 4342$ background and tested for their ability to rescue the NMJ growth defect. Postsynaptic expression of dCIP4L145D, but not HA-dCIP4-L436S and HA-dCIP4-W603K, rescued the synaptic overgrowth phenotype of dcip $4^{1} /$ Df(3L)ED4342 larvae (Fig. 2D-I; supplemental Table 1, available at www. jneurosci.org as supplemental material). The postsynaptic targeting and expression levels of the mutant transgenes were similar to those of wild-type HA-dCIP4 (Fig. $4 F-I$; supplemental Fig. 3, available at www.jneurosci.org as supplemental material). Overall, these data suggest that Cdc42- and Wsp-binding domains, but not the membrane-tubulating activity, of $\mathrm{dCIP} 4$ are required for its regulatory role in synaptic growth.

Cdc42 function is required for efficient localization of dCIP4, which in turn controls the postsynaptic localization of Wsp

Given the biochemical interaction between dCIP4 and active Cdc42 or Wsp in vitro, we tested whether these proteins co-

localize at the NMJ. Immunostaining of third instar larvae expressing Myc-tagged Cdc42V12 in the muscle with anti-dCIP4, anti-Myc, and anti-Wsp (Bogdan et al., 2005) revealed strong colocalization of dCIP4, Cdc42V12, and Wsp in the postsynaptic region of type I NMJs (Fig. 4A1--A4), suggesting the possibility that they can form complexes in vivo.

In the wing epithelium, $\mathrm{Cdc} 42$ has been shown to be required for the apical localization of dCIP4 (Fricke et al., 2009). To determine whether Cdc42 function is also required for the postsynaptic localization of dCIP4 in muscles, we analyzed NMJs of $c d c 42^{2}$, a hypomorphic allele of $c d c 42$ (Genova et al., 2000). We found a significant decrease in levels of postsynaptic dCIP4 (Fig. 4B1-C2; supplemental Fig. $4 A$, available at www.jneurosci.org as supplemental material). Levels of dCIP4 expression in the muscles of $c d c 42^{2} / c d c 42^{2}$ mutant larvae were normal (supplemental Fig. $4 B$, available at www.jneurosci.org as supplemental material), suggesting that $\mathrm{Cdc} 42$ function is required for efficient postsyn- aptic localization of dCIP4 at NMJs. In contrast, the postsynaptic localization of Myc-tagged Cdc42V12 expressed in muscles was normal in $d c i p 4^{1} / D f(3 L) E D 4342$ mutant larvae (data not shown).

We next examined the effect of dcip4 mutations on Wsp localization. In $d c i p 4^{1} / D f(3 L) E D 4342$ mutant larvae, the Wsp signal was almost completely absent from the NMJ postsynapse (Fig. 4D1--E2; supplemental Fig. 4A, available at www.jneurosci. org as supplemental material). Levels of Wsp expression appeared to be normal in $d c i p 4$ mutant muscles (supplemental Fig. $4 C$, available at www.jneurosci.org as supplemental material), suggesting that dCIP4 is required for the postsynaptic localization of Wsp. In contrast, the postsynaptic localization and abundance of dCIP4 in wsp null mutants [ $\left.w s p^{1} / D f(3 R) 3450\right]$ were normal (data not shown). To further characterize the role of $\mathrm{dCIP} 4$ in the postsynaptic localization of Wsp, we examined Wsp localization in $d c i p 4^{1} / D f(3 L) E D 4342$ mutant larvae expressing 

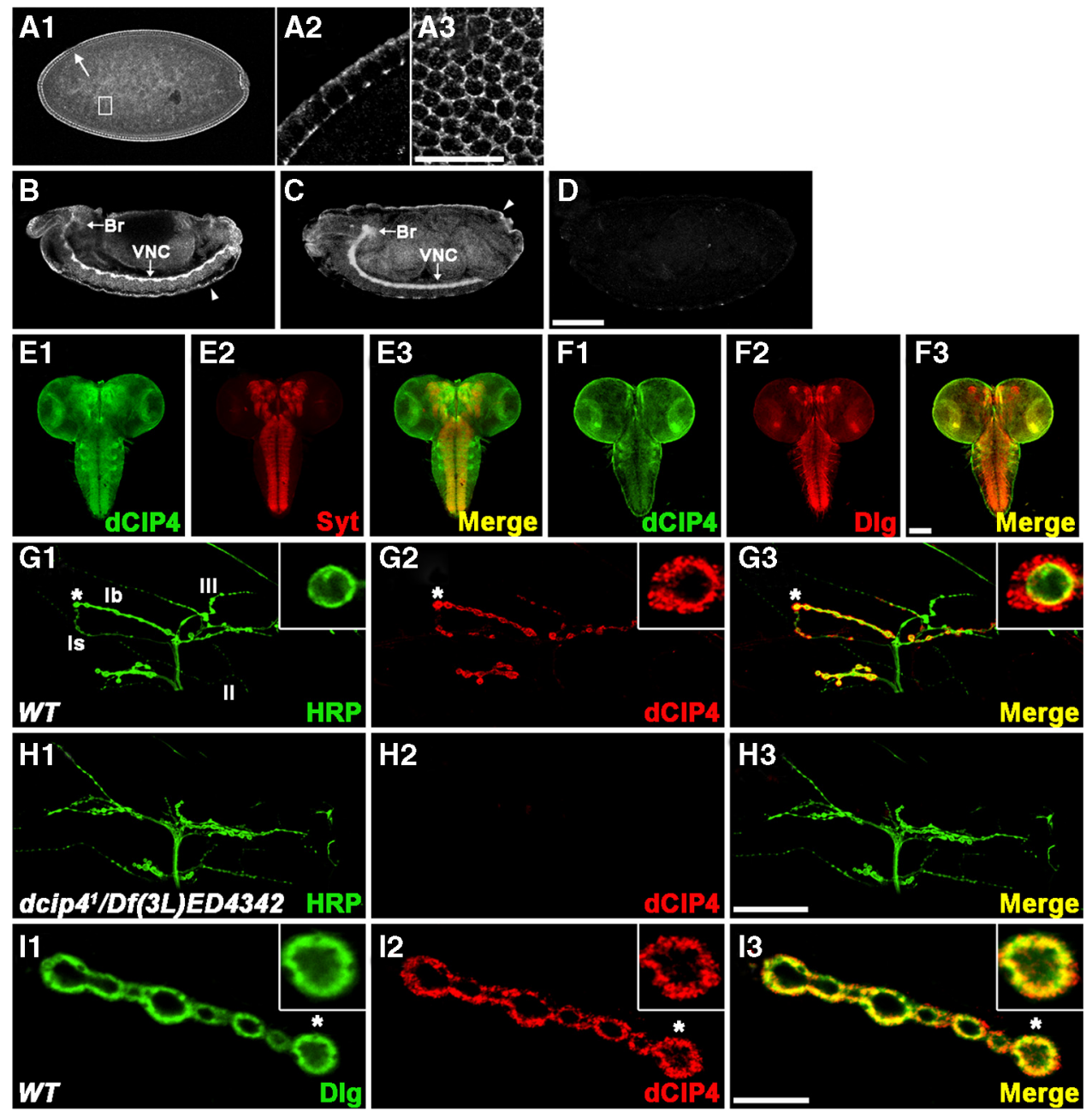

Figure 3. dCIP4 expression in the CNS and at the NMJ.A1-A3, Lateral view of a late stage 5 wild-type embryo stained with anti-dCIP4. Higher-magnification images of the areas indicated by the arrow and box in $\boldsymbol{A} \mathbf{1}$ are shown in $\boldsymbol{A} \mathbf{2}$ and $\boldsymbol{A} \mathbf{3}$, respectively. The image shown in $\boldsymbol{A} \mathbf{3}$ is a superficial optical section of an embryo. Note that the maternally derived dCIP4 protein is highly enriched at the cell borders. Dorsal is up, anterior is to the left. $\boldsymbol{B}, \boldsymbol{C}$, Lateral views of stage $13(\boldsymbol{B})$ and $16(\boldsymbol{C})$ wild-type embryos stained with anti-dCIP4. dCIP4 is highly concentrated in the brain (Br) and VNC and the surface epithelium (arrowheads). D, Lateral view of a stage $16 \mathrm{dcip4} 4^{1} / D f(3 L) E D 4342$ embryo stained with anti-dCIP4. E1-F3, Confocal images of the CNS dissected out of wild-type third instar larvae double stained with anti-dCIP4 (green) and either anti-Syt (red) or anti-Dlg (red). G1-H3, Confocal images of NMJs 12/13 double stained with anti-HRP (green) and anti-dCIP4 (red). In wild-type larvae (G1-G3), dCIP4 immunoreactivity is detected only at type lb and Is boutons and is absent at other types. Insets in G1-G3 show higher-magnification images of single Ib boutons labeled with asterisks. dcip4 ${ }^{1}$ IDf(3L)ED4342 mutant larvae (H1-H3) lack detectable dCIP4 staining at the NMJ 12/13. I1-I3, A single confocal slice of an NMJ 6/7 branch with type Ib boutons double stained with anti-Dlg (green) and anti-dCIP4 (red). Insets show higher-magnification images of single boutons labeled with asterisks. Scale bars: (in A3) A2, A3, $10 \mu \mathrm{m}$; (in D) A1, B-D, $100 \mu \mathrm{m}$;

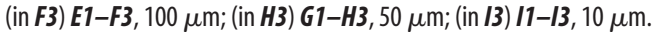

wild-type or mutant dCIP4 transgenes in muscle cells. Muscular expression of HA-dCIP4, HA-dCIP4-L145D, or HA-dCIP4L436S restored postsynaptic Wsp levels similar to wild-type, whereas HA-dCIP4-W603K had no such activity (Fig. 4F1-I3). Together, our data suggest that the dCIP4-Wsp interaction mediated by the SH3 domain of dCIP4 is necessary for Wsp localization to the NMJ postsynapse.

Postsynaptic Cdc42, Wsp, and Arp2/3 complex inhibit synaptic growth at the NMJ

Based on the biochemical and genetic data described above, we hypothesized that dCIP4 may act in the postsynaptic Cdc42-Wsp pathway to regulate synaptic growth. However, the role of postsynaptic Cdc42 in the regulation of synaptic growth at the NMJ has not been characterized. To test this directly, we examined the morphology of NMJs in larvae expressing $c d c 42$ dsRNA postsynaptically (Fig. 5; supplemental Table 2, available at www.jneurosci.org as supplemental material). In a control exper- iment, expression of $U A S-c d c 42^{R N A i}$ in neurons using C155GAL4 increased overall bouton number, satellite bouton number, and total NMJ length (Fig. $5 F-H$ ), confirming the previous finding that presynaptic $\mathrm{Cdc} 42$ functions to restrain synaptic growth (Rodal et al., 2008) and the efficacy of transgenic RNAi. When the same UAS transgene was expressed in muscles using BG57-GAL4, we observed an increase in all the parameters of synaptic growth in $U A S-c d c 42^{R N A i} /+$; BG57-GAL/+ compared with the $B G 57-G A L /+$ control larvae (Fig. $5 A, B, F--H$ ), supporting the role of postsynaptic Cdc42 in synaptic growth regulation. To confirm the regulatory role of postsynaptic Cdc42, we also examined NMJ morphology in wild-type larvae expressing the constitutively active mutant Cdc42V12 or the dominantnegative mutant Cdc42N17 in muscles only (supplemental Fig. 5, supplemental Table 3, available at www.jneurosci.org as supplemental material). Expression of $U A S-c d c 42 \mathrm{~V} 12$ driven by $\mathrm{MHC}$ GS-GAL4 in the absence of RU486 (Osterwalder et al., 2001) was sufficient to decrease all parameters of synaptic growth, whereas 

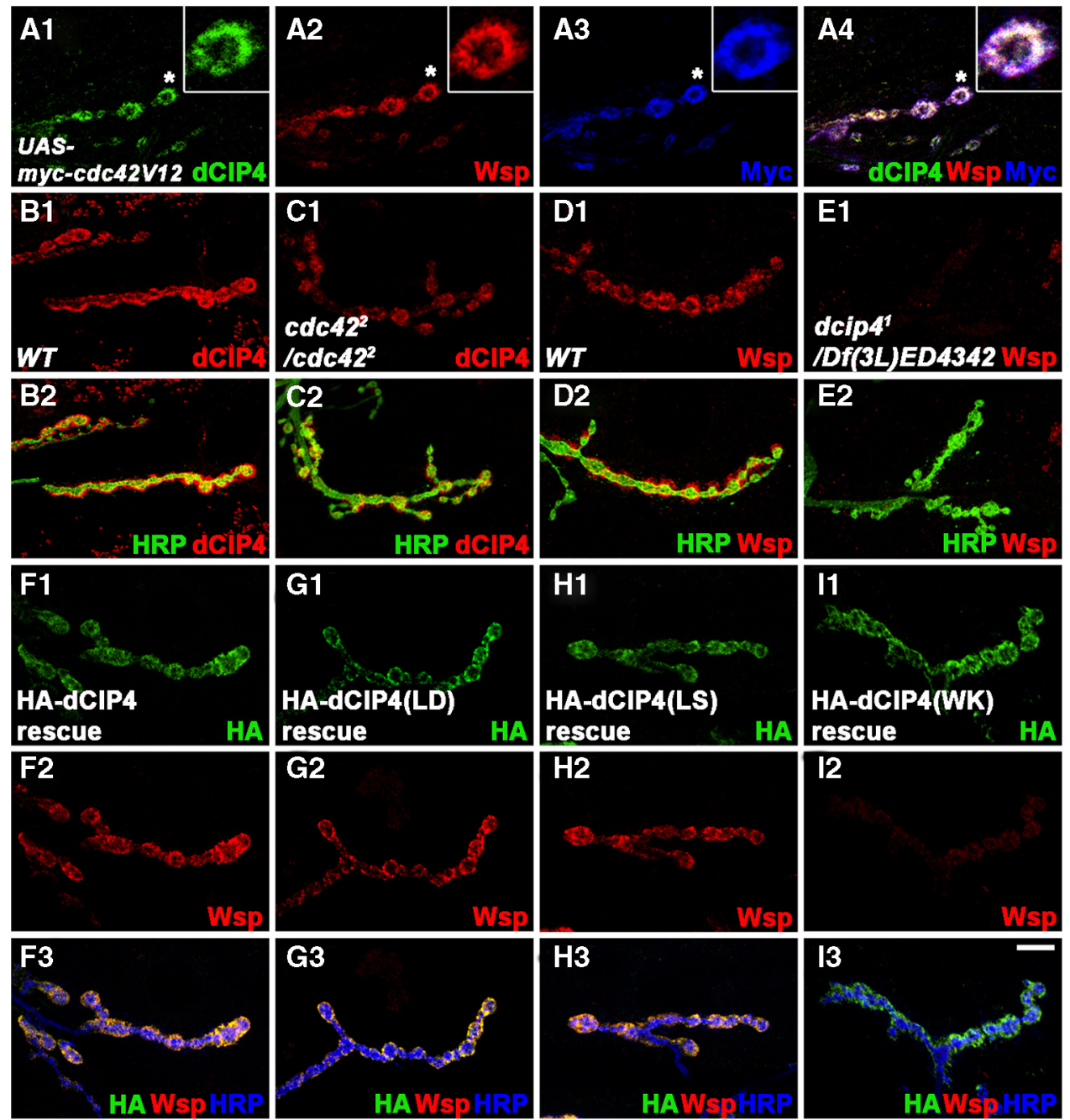

Figure 4. dCIP4 controls postsynaptic Wsp localization. A1-A4, A single confocal slice of an NMJ 6/7 triple stained with anti-dCIP4 (green), anti-Wsp (red), and anti-Myc (blue) is shown for wild-type $\left(\right.$ WT; $w^{1118}$ ) larvae overexpressing Myc-tagged Cdc42V12 in muscles. Insets show higher-magnification images of single lb boutons labeled with asterisks. B1-C2, Single confocal slices of NMJs 6/7 double stained with anti-dCIP4 (red) and anti-HRP (green) are shown for wild-type ( $y w ; B 1, B 2$ ) and $c d c 42^{2} / c d c 42^{2}$ mutant (C1, C2) larvae. D1-E2, Single confocal slices of NMJs 6/7 double stained with anti-Wsp (red) and anti-HRP (green) are shown for wild-type $\left(w^{1118} ; \mathbf{D 1}, \mathbf{D 2}\right)$ and dcip4 $/$ Df(3L)ED4342 mutant (E1, E2) larvae. F1-I3, Single confocal slices of NMJs 6/7 triple stained with anti-HA (green), anti-Wsp (red), and anti-HRP (blue) are shown for dcip4 ${ }^{1} / D f(3 L) E D 4342$ mutant larvae postsynaptically expressing wild-type HA-dCIP4 (F1-F3), HA-dCIP4-L145D [dCIP4(LD); G1-G3], HA-dCIP4-L436S [dCIP4(LS); H1-H3], HA-dCIP4-W603K [dCIP4(WK); I1-I3]. Postsynaptic expression of wild-type dCIP4, dCIP4-L145D, or dCIP4-L436S restores normal postsynaptic localization of endogenous Wsp in dcip4 $4^{7}$ I ff(3L)ED4342 mutant larvae, whereas dCIP4-W603K fails to do so. Scale bar, $10 \mu \mathrm{m}$.

leaky UAS-cdc42N17 expression had the opposite effects (supplemental Fig. 5A-F, available at www.jneurosci.org as supplemental material). Thus, our results suggest that levels of postsynaptic Cdc42 signaling are inversely correlated to the extent of synaptic growth at the NMJ.

Next, we investigated the possible role of postsynaptic Wsp in synaptic growth regulation. For this purpose, we expressed a wsp dsRNA using the UAS/GAL4 system. Expression of a UAS$w s p^{R N A i}$ transgene in muscles using BG57-GAL4 significantly increased bouton number, satellite bouton number, and total NMJ length $(p<0.001)$ (Fig. 5C,F--H; supplemental Table 2, available at www.jneurosci.org as supplemental material), recapitulating the synaptic overgrowth phenotype of $w s p$ loss-of-function mutants (Coyle et al., 2004; Rodal et al., 2008). However, expression of the same RNAi transgene in neurons using C155-GAL4 had no significant effects on synaptic growth (supplemental Table 2, available at www.jneurosci.org as supplemental material). Because the UAS-wsp dsRNA approach does not completely eliminate Wsp immunoreactivity at the larval NMJ (data not shown), we further evaluated the tissue-specific requirement of Wsp by expressing exogenous Wsp in a wsp null-mutant background [ $\left.w s p^{1} / D f(3 R) 3450\right]$ using the UAS/GAL4 system. Muscular expression of UAS-wsp in $w s p^{1} / D f(3 R) 3450$ larvae using BG57GAL4 substantially rescued the increase in all parameters of synaptic growth $(p<0.001)$ (supplemental Fig. 5G-I, supplemental Table 3, available at www.jneurosci.org as supplemental material). Neuronal expression of UAS-wsp using C155-GAL also rescued the increase in satellite bouton number, but not the increase in overall bouton number and NMJ length (supplemental Fig. 5G-I, available at www.jneurosci.org as supplemental material). Thus, our data support the notion that Wsp is required both pre-and postsynaptically for normal synaptic growth.

Finally, we evaluated whether the Arp2/3 complex, the effector of Wsp, also functions postsynaptically to regulate synaptic growth. Muscle-specific expression of UAS-arp $2^{R N A i}$ or UAS$\operatorname{arp} 3^{R N A i}$ using BG57-GAL4 caused synaptic overgrowth (Fig. $5 D-H$; supplemental Table 2, available at www.jneurosci.org as supplemental material), revealing the involvement of the 

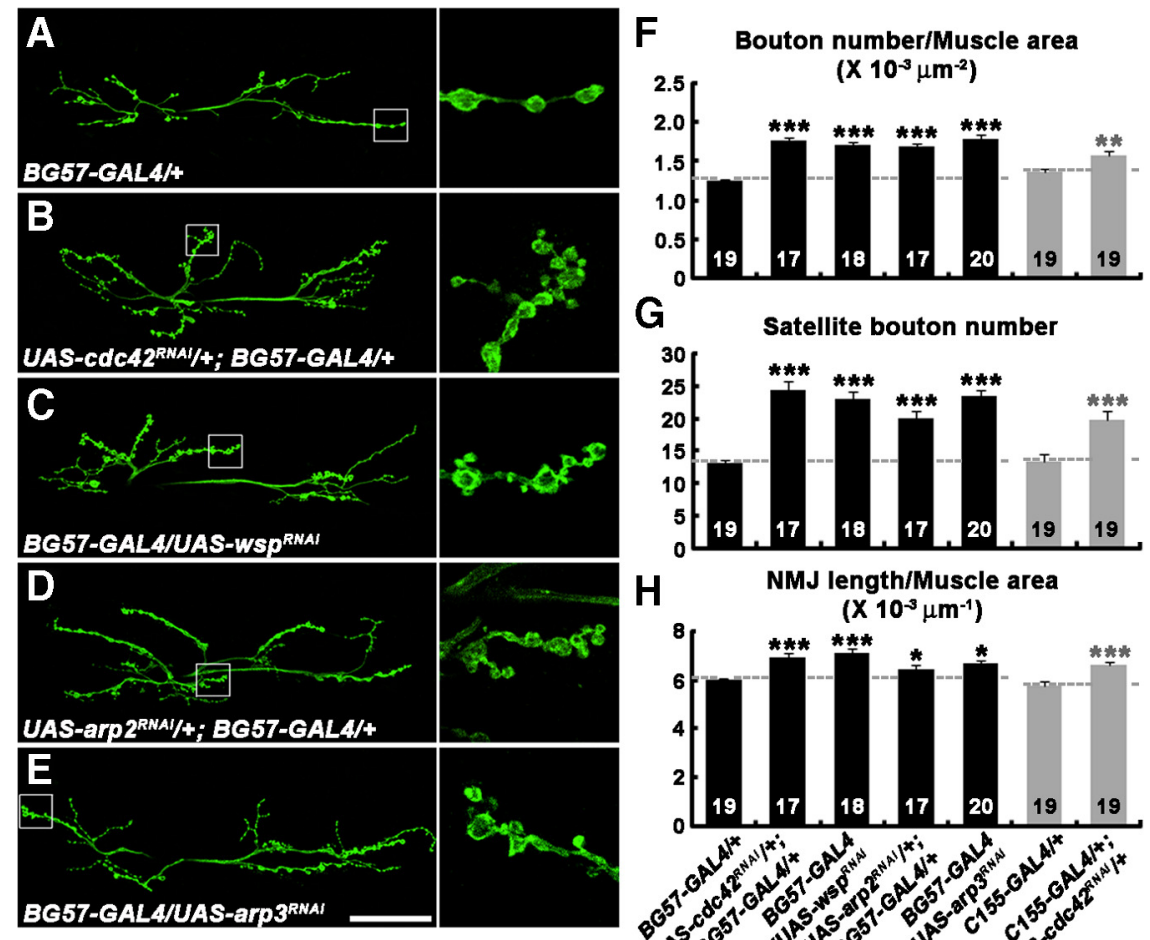

\section{G}

Satellite bouton number

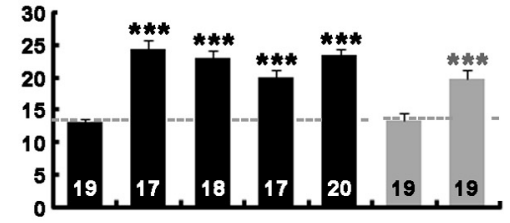

$\mathrm{H}$

NMJ length/Muscle area $\left(\mathrm{X} 10^{-3} \mu \mathrm{m}^{-1}\right)$

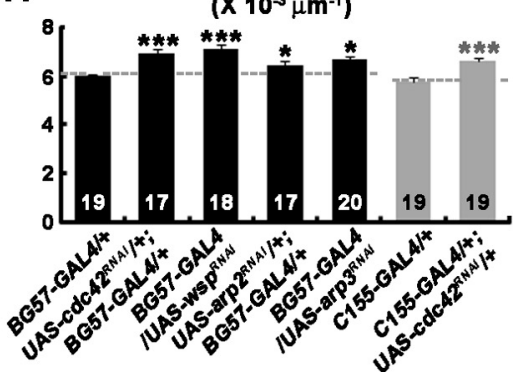

Figure 5. Postsynaptic Cdc42, Wsp, and the Arp2/3 complex regulate synaptic growth at the NMJ. $\boldsymbol{A}-\boldsymbol{E}$, Confocal images of

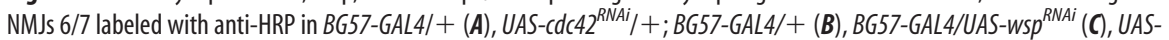
$\operatorname{arp}^{R N A i} /+; B G 57-G A L 4 /+(\boldsymbol{D})$, and BG57-GAL4/UAS-arp3 ${ }^{R N A i}(\boldsymbol{E})$ third instar larvae. The columns on the right are highermagnification views of NMJ terminals marked in the low-magnification panels by boxes. Note that when expressed specifically in muscles, cdc42, wsp, arp2, and arp3 dsRNAs increase satellite bouton formation similarly. Scale bar, $50 \mu \mathrm{m}$. $\boldsymbol{F}$ - $\boldsymbol{H}$, Quantification of total bouton number $(\boldsymbol{F})$, satellite bouton number $(\boldsymbol{G})$, and total NMJ length $(\boldsymbol{H})$ at NMJ 6/7 in the genotypes indicated. The number of NMJs quantified for each genotype is indicated inside the bars. Comparisons are with the control BG57-GAL4/+ or C155-GAL4/+ line $\left({ }^{*} p<0.05 ;{ }^{* *} p<0.01 ;{ }^{* * *} p<0.001\right)$. Raw data are shown in supplemental Table 2 (available at www.jneurosci.org as supplemental material).

postsynaptic Arp2/3 complex in the regulation of synaptic growth at the NMJ.

dcip4 interacts with the Cdc42-Wsp pathway at the NMJ and regulates postsynaptic F-actin

To further test the possibility that dCIP4, Cdc42, Wsp, and the Arp2/3 complex function in a common pathway at the NMJ, we examined transheterozygous interactions between dcip 4 and cdc42, wsp, or arp3 (Fig. 6; supplemental Table 4, available at www.jneurosci.org as supplemental material). Heterozygosity for $d c i p 4^{1}, w s p^{1}$, or $\operatorname{arp} 3^{E P 3640}$, a strong hypomorphic P-element allele of arp3 (Hudson and Cooley, 2002), did not change any NMJ synaptic parameter (Fig. 6E-G). In larvae heterozygous for $c d c 42^{2}$, a mild hypomorphic allele of $c d c 42$ (Genova et al., 2000), total bouton number and NMJ length were normal, but satellite bouton formation was slightly increased (Fig. $6 E-G$ ). All of these parameters of synaptic growth were increased in double heterozygotes (i.e., $c d c 42^{2} /+$; $d c i p 4^{1} /+, d c i p 4^{1},+/+, w s p^{1}$, and $d c i p 4^{1},+/+$, $\operatorname{arp} 3^{E P 3640}$ ) compared with single heterozygotes for $d c i p 4, c d c 42$, wsp, or arp3 alone (Fig. $6 A-C, E--G$ ). The number of satellite boutons was further increased in larvae transheterozygous for $d c i p 4, c d c 42$, and wsp (Fig. 6D,F). These observations strongly support the model in which dCIP4 acts in the Cdc42-WspArp2/3 pathway to restrain synaptic growth.

This conclusion led us to examine whether dCIP4 plays a critical role in the regulation of actin dynamics at NMJs. In wildtype larvae, F-actin visualized by rhodamine-conjugated pha-

lloidin was highly enriched in the postsynaptic side of NMJs (supplemental Fig. $6 A 1, A 2$, available at www.jneurosci.org as supplemental material), as described previously (Coyle et al., 2004). Levels of postsynaptic F-actin were significantly reduced in $\operatorname{dcip}^{1} / \mathrm{Df}(3 \mathrm{~L})$ ED4342 compared with wild-type larvae $(p<0.01)$ (supplemental Fig. 6B1,B2,F, available at www. jneurosci.org as supplemental material), supporting the involvement of dCIP4 in postsynaptic F-actin regulation. A similar phenotype was observed in $c d c 42, w s p$, or arp3 mutants (supplemental Fig. 6C1--F, available at www.jneurosci.org as supplemental material). These results, together with a recent report that $\mathrm{dCIP} 4$ activates Wsp-Arp2/3-mediated actin polymerization in vitro (Fricke et al., 2009), suggest that dCIP4 acts in the Cdc42-WspArp2/3 pathway to regulate postsynaptic F-actin.

\section{Postsynaptic dCIP4 and Wsp restrain} synaptic growth by attenuating presynaptic BMP signaling

Mutations activating retrograde BMP signaling lead to an increase in total bouton number and satellite bouton formation (O'Connor-Giles et al., 2008). Because these phenotypes are very similar to those observed in $d c i p 4, c d c 42, w s p$, and $\operatorname{arp} 3$, we wondered whether the signaling cascade involving postsynaptic dCIP4, Cdc42, Wsp, and Arp2/3 complex might negatively regulate retrograde BMP signaling. Accumulation of P-Mad at the terminals and in the nuclei of motor neurons is indicative of retrograde BMP signaling at the NMJ (Marques et al., 2002; McCabe et al., 2003). Thus, we examined levels of P-Mad levels in larvae expressing UAS-dcip $4^{R N A i}$, $U A S-c d c 42^{R N A i}, U A S-w s p^{R N A i}, U A S-\operatorname{arp} 2^{R N A i}$, or UAS-arp $3^{R N A i}$ under the control of BG57-GAL4. In all these animals, P-Mad levels at the terminals and in the nuclei of motor neurons were significantly increased compared with controls $(p<0.001)$ (Fig. $7 A-G$; supplemental Fig. 7, available at www.jneurosci.org as supplemental material).

If postsynaptic dCIP4 and Wsp restrain synaptic growth by attenuating retrograde BMP signaling, synaptic overgrowth caused by knockdown of postsynaptic dCIP4 or Wsp would be affected by additional mutations in whishful thinking (wit), a gene encoding a type-II BMP receptor (Aberle et al., 2002). After confirming synaptic undergrowth in $w i t^{A 12} / w_{i t}{ }^{B 11}$, we analyzed NMJ morphology in wit $^{A 12} /$ wit $^{B 11}$ larvae expressing either UAS$d c i p 4^{R N A i}$ or $U A S-w s p^{R N A i}$ postsynaptically. Overall bouton number and satellite bouton formation in these larvae were not significantly different from those in $w i t^{A 12} / w_{i t}^{B 11}$ larvae $(p>0.1)$ (Fig. $7 H$ ), suggesting that synaptic overgrowth induced by knockdown of postsynaptic dCIP4 or Wsp requires BMP signaling through Wit. We also found that heterozygosity for wit $\left(\right.$ wit $\left.^{\mathrm{Al2} /}+\right)$ could completely reverse the increase in total bouton number and satellite bouton formation in larvae expressing either UAS-dcip $4^{R N A i}$ or $U A S-w s p^{R N A i}$ postsynaptically (Fig. $7 \mathrm{H}$ ). In a control experiment, heterozygosity for wit did not cause any 

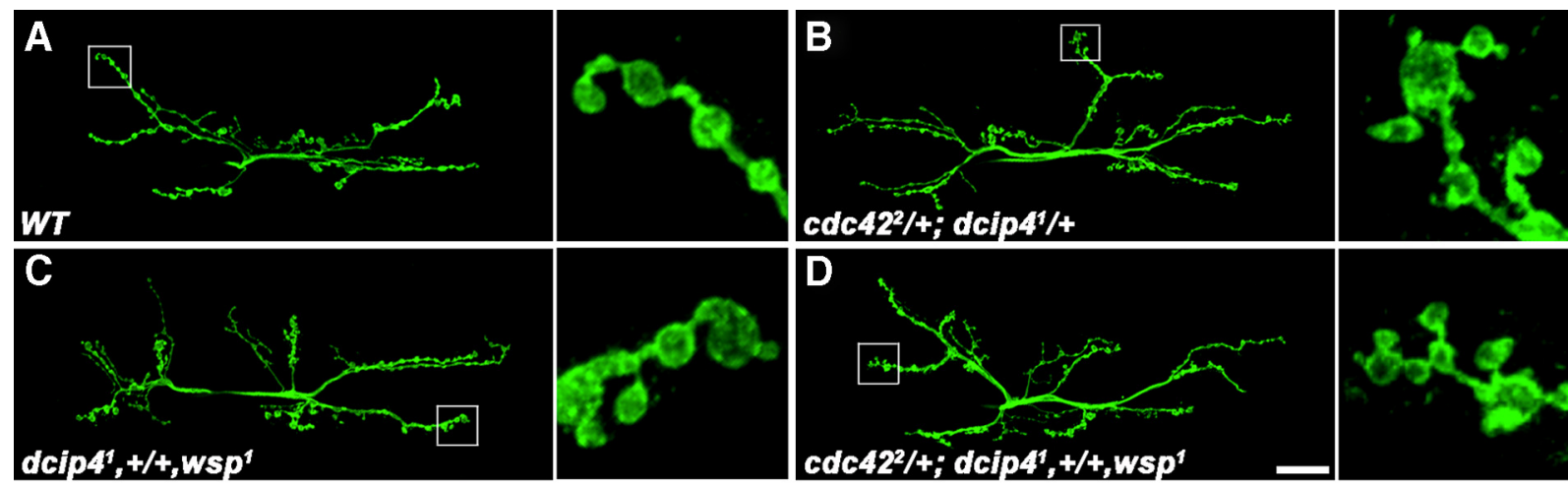

$\mathbf{E}$

$\mathbf{F}$

Bouton number/Muscle area $\left(\mathrm{X} 10^{-3} \mu \mathrm{m}^{-2}\right)$

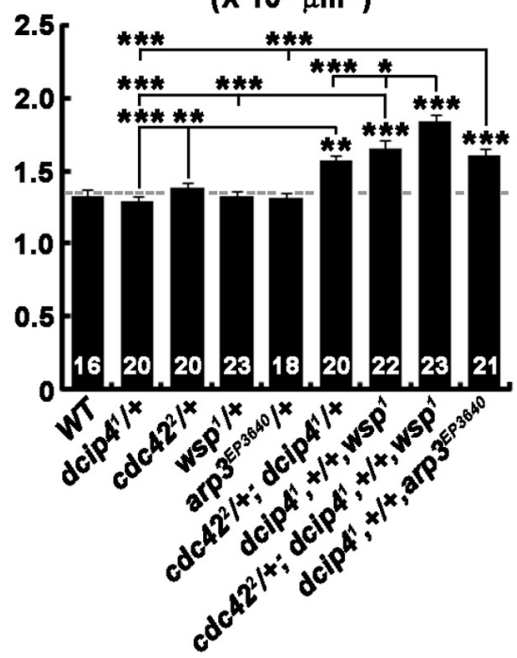

Satellite bouton number

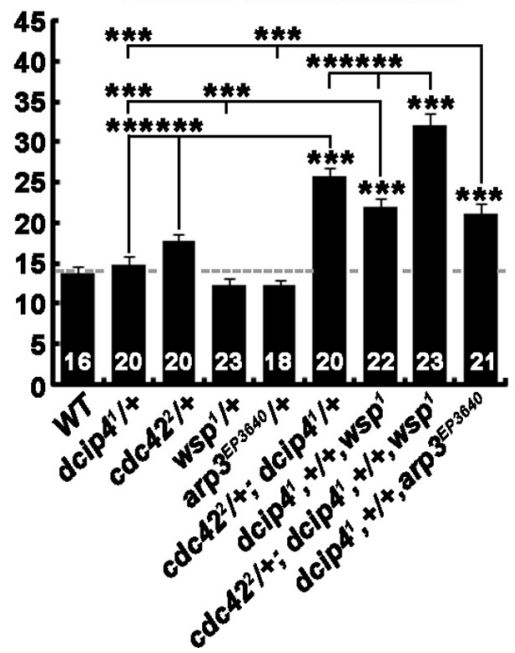

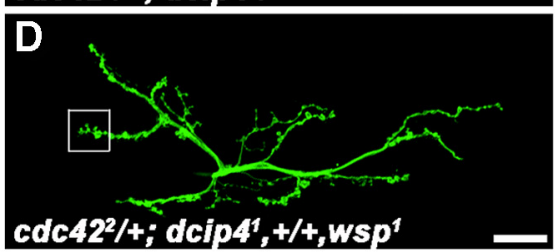

G

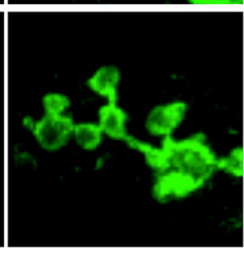

NMJ length/Muscle area (X 10 $\left.{ }^{-3} \mu \mathrm{m}^{-1}\right)$

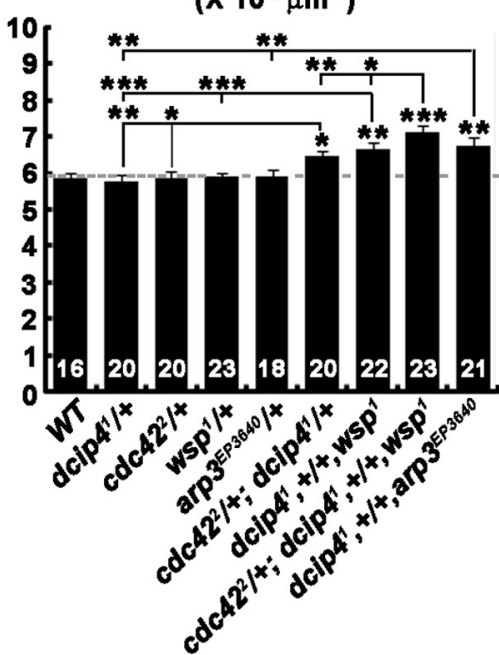

Figure 6. dcip4 interacts with $c d c 42$, wsp, and arp3. A-D, Confocal images of NMJs $6 / 7$ labeled with anti-HRP in wild-type (WT; $\boldsymbol{A}), c d c 42^{2} /+; d c i p 4^{1} /+(\boldsymbol{B}), d c i p 4^{1},+/+$, wsp ${ }^{1}(\boldsymbol{C})$, and $c d c 42^{2} /+; d c i p 4^{7},+/+, w s p^{1}(\boldsymbol{D})$ third instar larvae. Columns to the right side of $\boldsymbol{A}-\boldsymbol{D}$ are higher-magnification views of NMJ terminals marked in the low-magnification panels by boxes. Compared to wild-type, $c d c 42^{2} /+; d c i p 4^{1} /+, d c i p 4^{1},+/+$, wsp ${ }^{1}$, and $c d c 42^{2} /+; d c i p 4^{1},+/+$, wsp $p^{7}$ larvae exhibit a significant increase in satellite bouton formation. Scale bar, $25 \mu \mathrm{m}$. E-G, Quantification of total bouton number $(\boldsymbol{E})$, satellite bouton number $(\boldsymbol{F})$, and total NMJ length $(\boldsymbol{G})$ at NMJ 6/7 in the genotypes indicated. The number of NMJs quantified for each genotype is indicated inside the bars. All comparisons are with wild-type unless indicated $\left({ }^{*} p<0.05 ;{ }^{* *} p<0.01 ;{ }^{* * *} p<0.001\right.$ ). Raw data are shown in supplemental Table 4 (available at www.jneurosci.org as supplemental material).

synaptic growth defect, suggesting that postsynaptic dCIP4 and Wsp restrain synaptic growth by attenuating retrograde BMP signaling.

\section{dCIP4/Wsp inhibits postsynaptic Gbb secretion}

To investigate the mechanism by which the postsynaptic dCIP4Wsp pathway regulates the retrograde Gbb signal, we generated a transgene harboring UAS-gbb-GFP. Muscular expression of $U A S$ - $g b b$-GFP using BG57-GAL4 led to a significant increase in presynaptic $\mathrm{P}$-Mad levels and satellite bouton formation (supplemental Fig. $8 A-C$, available at www.jneurosci.org as supplemental material), suggesting that the expressed Gbb-GFP fusion protein is biologically active. Gbb-GFP was produced as a precursor protein of $\sim 75 \mathrm{kDa}$ ( $48 \mathrm{kDa}$ from the unprocessed Gbb plus $27 \mathrm{kDa}$ from GFP) that was processed to a smaller protein of $\sim 44$ $\mathrm{kDa}$ (17 kDa from the processed Gbb plus $27 \mathrm{kDa}$ from GFP) (supplemental Fig. $8 D$, available at www.jneurosci.org as supplemental material). Thus, the Gbb-GFP fusion protein was processed as expected (Doctor et al., 1992).

We then analyzed the effect of dcip4 mutations and postsynaptic wsp RNAi on the levels of extracellular Gbb-GFP at the NMJ. In this experiment, third instar larval fillets were incubated with an anti-GFP antibody before fixation, as described previ- ously for the selective detection of extracellular Wg (Strigini and Cohen, 2000). The extracellular GFP signal at NMJs was significantly increased in BG57-GAL4,Df(3L)ED4342/UAS-gbbGFP,dcip $4^{1}$ and BG57-GAL4/UAS-gbb-GFP,UAS-wsp ${ }^{R N A i}$ larvae compared with the BG57-GAL4/UAS-gbb-GFP control $(p<$ 0.01 ) (Fig. $8 B-E$ ). GFP immunoreactivity was significantly decreased in BG57-GAL4/+ compared with BG57-GAL4/UAS-gbbGFP larvae (Fig. 8, compare $A, B$ ), confirming the specificity of GFP staining. The expression level and processing of Gbb-GFP were not significantly altered by loss of postsynaptic dcip 4 and wsp function (supplemental Fig. $8 D-H$, available at www. jneurosci.org as supplemental material), suggesting that postsynaptic dCIP4 and Wsp may be required for Gbb secretion and/or endocytosis.

In mammals, dynamin-dependent endocytosis plays a crucial role in the internalization of ionotropic glutamate receptors from the postsynaptic plasma membrane (Carroll et al., 1999). Consistent with this, the synaptic abundance of GluRIIA and GluRIIB was increased in a temperature-sensitive dynamin mutant $\left(s h i^{t s}\right)$, which was pulsed at restrictive temperature (supplemental Fig. $9 E$, available at www.jneurosci.org as supplemental material). A similar phenotype was observed in larvae expressing a dominant negative form of dynamin (Shi ${ }^{\mathrm{K} 44 \mathrm{~A}}$ ) (Moline et al., 1999) only in 

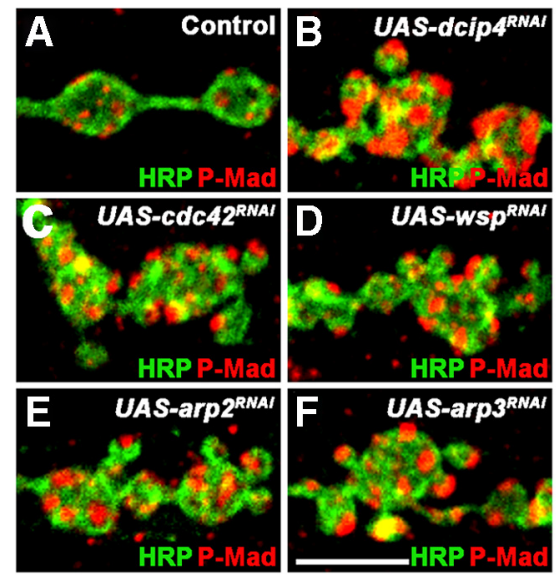

G

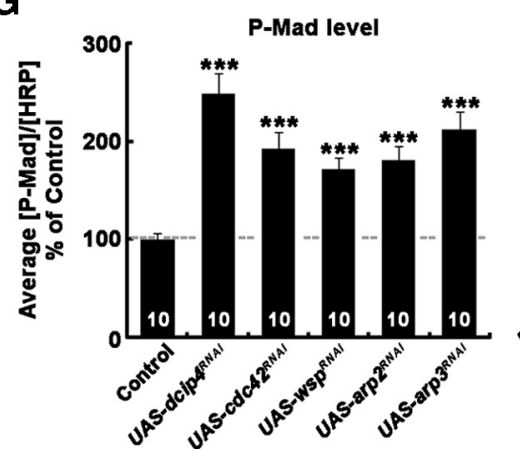

\section{$\mathrm{H}$}

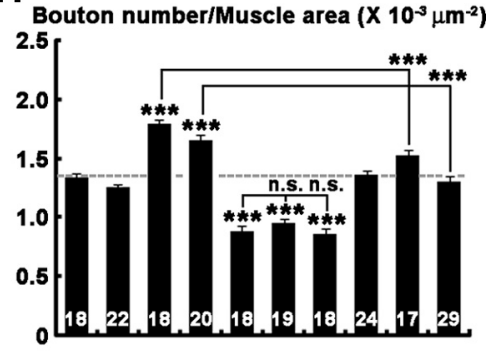

Satellite bouton number

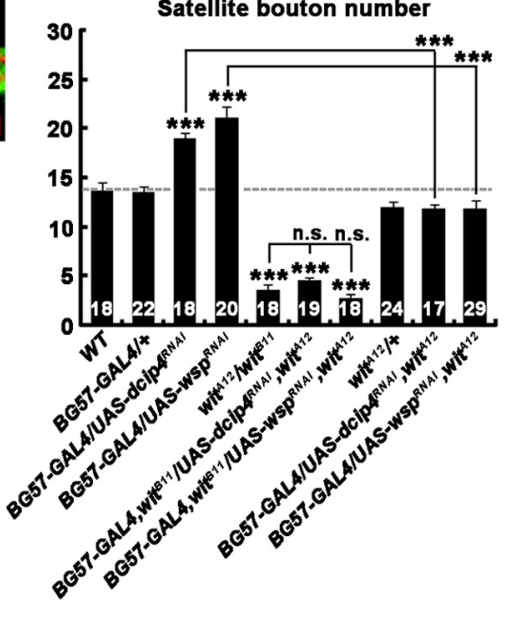

Figure 7. Postsynaptic dCIP4, Cdc42, Wsp, and the Arp2/3 complex attenuate presynaptic BMP signaling. $\boldsymbol{A}-\boldsymbol{F}$, Highmagnification images of Ib boutons at NMJs $6 / 7$ labeled with anti-P-Mad (red) and anti-HRP (green) in control (BG57-GAL4/+;A), BG57-GAL4/UAS-dcip4 ${ }^{\text {RNAi }}(\boldsymbol{B})$, UAS-cdC42 $2^{\text {RNAi }} /+;$ BG57-GAL4I+ (C), BG57-GAL4/UAS-WSp ${ }^{\text {RNAi }}(\boldsymbol{D})$, UAS-arp2 ${ }^{\text {RNAi } /+; ~ B G 57-~}$ $G A L 4 /+(\boldsymbol{E})$, and BG57-GAL4/UAS-arp ${ }^{R N A i}(\boldsymbol{F})$ larvae. Note that presynaptic levels of P-Mad are significantly increased by reduction of postsynapticdcip4, cdc42, wsp, arp2, or arp3 activity. Scale bar, $5 \mu \mathrm{m}$. G, Quantification of the ratio of average P-Mad to HRP levels in larvae of the genotypes indicated. Values are percentages of the BG57-GAL4/+ control. $\boldsymbol{H}$, Quantification of overall bouton number and satellite bouton formation at NMJ 6/7 were quantified in the indicated genotypes. Note that synaptic overgrowth induced by dcip 4 or wsp RNAi is suppressed by reduction or loss of wit function. All comparisons are with wild-type (WT) unless indicated. n.s., Not significant. ${ }^{* *} p<0.001$. Raw data are shown in supplemental Table 5 (available at www.jneurosci.org as supplemental material).

muscles (supplemental Fig. 9A1--E, available at www.jneurosci.org as supplemental material), confirming the efficacy of $\mathrm{Shi}^{\mathrm{K} 44 \mathrm{~A}}$. To further investigate the mechanism of Gbb-GFP accumulation, we first analyzed larvae expressing both Gbb-GFP and Shi ${ }^{\mathrm{K} 44 \mathrm{~A}}$ in muscles. Levels of extracellular Gbb-GFP in these larvae were not significantly different from those in larvae with postsynaptic expression of Gbb-GFP alone ( $p=0.33$ ) (Fig. $8 E$ ), suggesting that the accumulation of extracellular Gbb-GFP at NMJs in larvae lacking postsynaptic dCIP4 or Wsp may not be attributable to dynamin-dependent endocytic defects. Next, we investigated the roles of dCIP 4 , Wsp, and the Arp $2 / 3$ complex in Gbb secretion. For this purpose, we transiently expressed the Gbb-GFP fusion protein in Drosophila S2R + cells and evaluated the effect of depleting dCIP4, Wsp, or Arp3 on the secretion of Gbb-GFP. The medium of untreated control cells contained only processed Gbb-GFP, whereas lysates of the same cells displayed processed and unprocessed Gbb-GFP (Fig. $8 F$ ). RNAi-mediated knockdown of dCIP4, Wsp, or Arp3 significantly increased the levels of processed Gbb-GFP in the medium normalized to those of intracellular Gbb-GFP (Fig. 8F,H). However, knockdown of dynamin did not affect the levels of processed Gbb-GFP in the medium (Fig. 8F,H), suggesting that defects in dynamindependent endocytosis do not account for the accumulation of processed Gbb-GFP in the medium. Finally, overexpression of
dCIP4 or Wsp resulted in a significant decrease in Gbb-GFP secretion (Fig. 8G,H). Together, our results support an inhibitory role for dCIP4 and Wsp in Gbb secretion.

\section{Discussion}

Neurons and muscles communicate through various transsynaptic signals to coordinate the development and remodeling of their synaptic terminals. At the Drosophila NMJ, the BMP ligand Gbb, secreted from postsynaptic muscles, acts as a critical retrograde signal that promotes the structural growth of presynaptic terminals. Previous studies have demonstrated that endocytic regulation of $\mathrm{BMP}$ receptors is a presynaptic mechanism to restrain Gbb-induced synaptic growth (Wang et al., 2007; O'ConnorGiles et al., 2008). Here, we present data demonstrating that inhibition of Gbb secretion by the postsynaptic dCIP4 pathway also contributes to fine regulation of retrograde BMP signaling and synaptic growth.

dCIP4 acts in the postsynaptic Cdc42Wsp-Arp2/3 pathway to negatively regulate synaptic growth

We have established a novel role for dCIP4 in synaptic growth regulation at the NMJ. Mutations in dcip4 cause synaptic overgrowth, as evidenced by an increase in overall bouton number, satellite bouton formation, and NMJ length. This phenotype is rescued by muscular but not neuronal expression of wild-type dCIP4. In addition, dCIP4 is highly enriched in the postsynaptic side of type I larval NMJs. Thus, we conclude that dCIP4 functions postsynaptically as a negative regulator of synaptic growth.

Our data demonstrate that dCIP4 acts positively in the postsynaptic Cdc42-Wsp-Arp2/3 pathway to restrain synaptic growth. First, we show that dCIP4 is able to form a multiprotein complex containing both active Cdc42 and Wsp in vitro. Second, a dCIP4 mutant defective in either Cdc42 or Wsp binding is not able to rescue the synaptic overgrowth phenotype in a dcip4 mutant background, suggesting a functional relationship between dCIP4 and the Cdc42-Wsp pathway. Third, postsynaptic knockdown of Cdc42, Wsp, or Arp3 causes similar NMJ phenotypes to those observed in dcip4 mutants. Fourth, dcip 4 displays transheterozygous interactions with $c d c 42, w s p$, and $\operatorname{arp} 3$ at the NMJ, thus establishing in vivo that these genes act in the same pathway to regulate synaptic growth. Fifth, dcip4 mutations, like wsp mutations, reduce postsynaptic F-actin at the NMJ, and dCIP4 function is required for the postsynaptic localization of Wsp. Finally, loss of Cdc42 function significantly impairs the postsynaptic localization of dCIP4, suggesting that dCIP4 acts as a Cdc42 effector at the NMJ. Together, our results suggest that dCIP4 acts downstream of Cdc42 to activate Wsp-Arp2/3-mediated actin polymerization in the NMJ postsynapse and that this activity of dCIP4 restrains synaptic growth. 


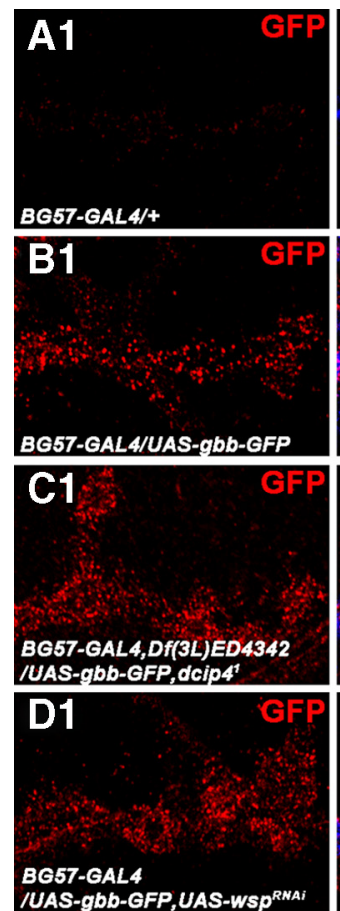

$\mathbf{E}$

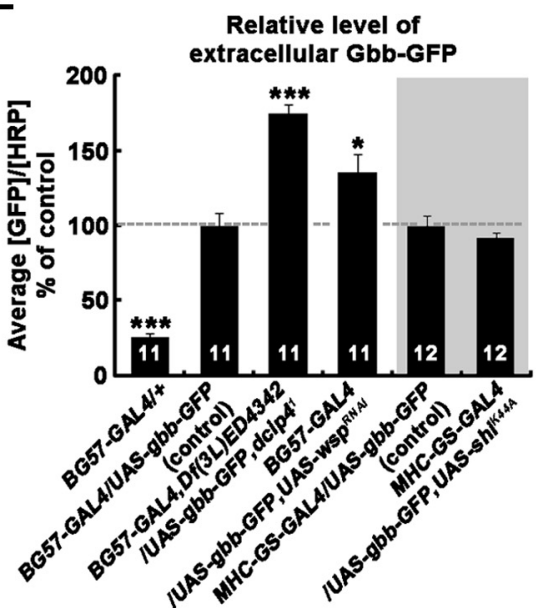

$\mathbf{F}$
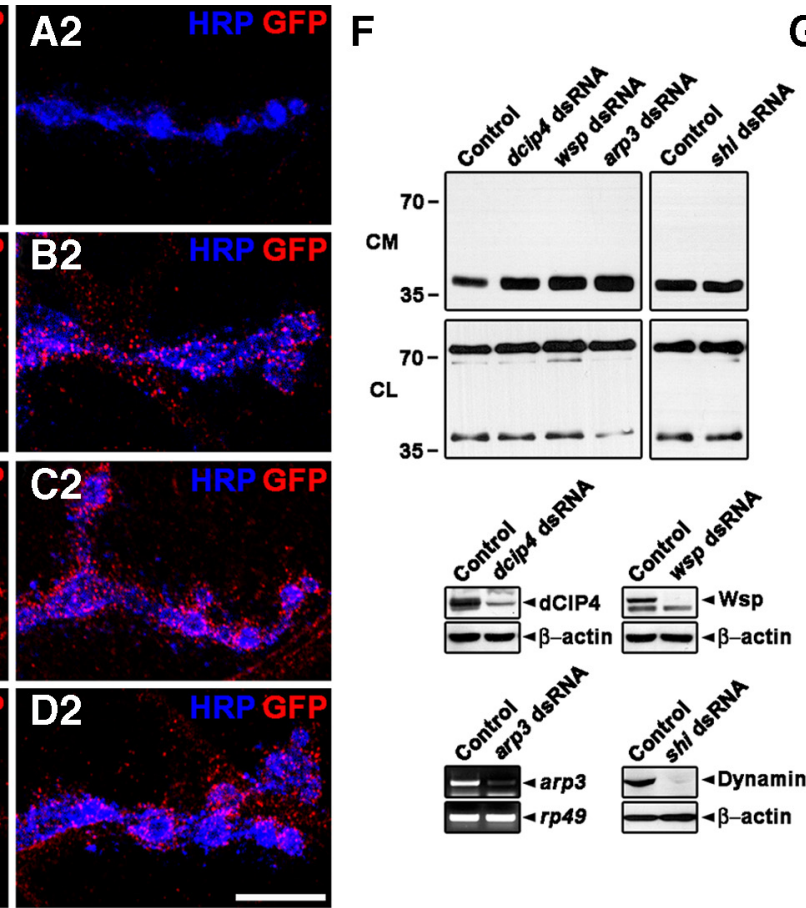

H
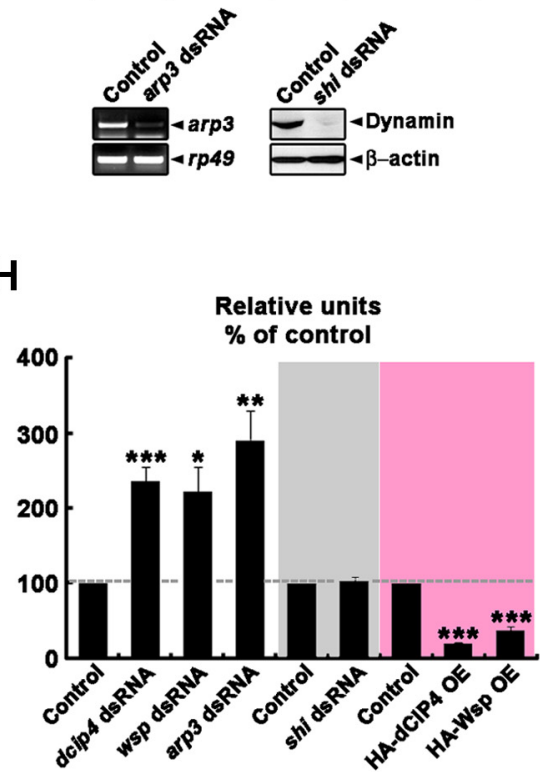

.
G
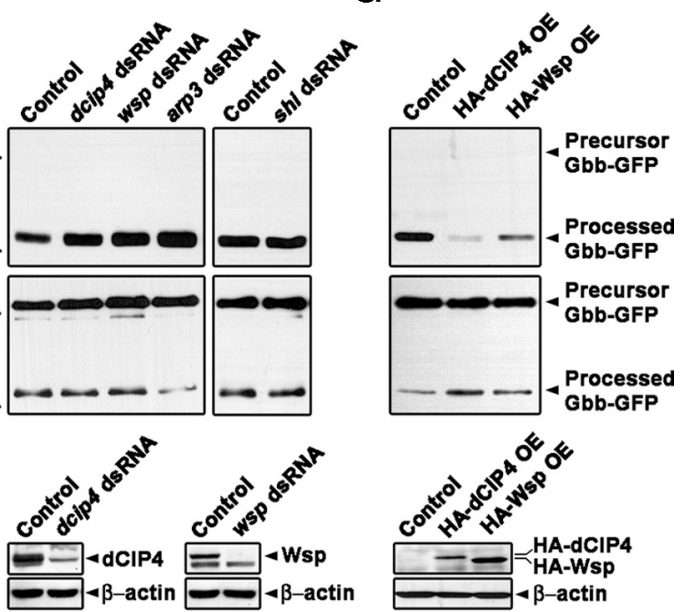

.

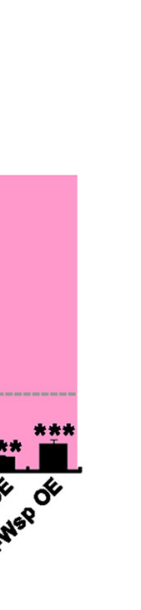

Figure 8. dCIP4 and Wsp inhibit Gbb secretion. A1-D2, Confocal images of NMJs 6/7 stained for extracellular Gbb-GFP (red) and HRP (blue) in BG57-GAL4/ + (A1, A2), BG57-GAL4/UAS-gbb-GFP (B1, B2), BG57-GAL4,Df(3L)ED4342/UAS-gbb-GFP, dcip4 ${ }^{\top}$ (C1, C2), and BG57-GAL4/UAS-gbb-GFP,UAS-wsp ${ }^{\text {RNAi }}$ (D1, D2) third instar larvae. Scale bar, $10 \mu \mathrm{m}$. E, Quantification of the ratio of average extracellular Gbb-GFP to HRP levels at NMJ 6/7 in third instar larvae of the genotypes indicated. All animals were raised on RU486-free medium. Values are percentages of the corresponding control. $\boldsymbol{F}-\boldsymbol{H}$, Effects of altering levels of dCIP4, Wsp, Arp3, or dynamin on the secretion of Gbb-GFP from S2R + cells. In F, GFP-containing proteins in the conditioned medium (CM) and lysates (CL) of S2R + cells transfected with Gbb-GFP and dsRNA against dcip4, wsp, arp3, or shi were detected by Western blot analysis using anti-GFP (see Materials and Methods). The efficacy and specificity of the dsRNAs were determined by Western blot (dcip4, wsp, and shidsRNAs) or RT-PCR (arp3 dsRNA) analysis. Levels of $\beta$-actin protein or rp49 mRNA were determined to ensure equal amounts of starting material. In G, GFP-containing proteins in the conditioned medium and lysates of S2R + cells expressing both Gbb-GFP and HA-dCIP4 or HA-Wsp were detected by Western blot analysis using anti-GFP (see Materials and Methods). Levels of HA-dCIP4 or HA-Wsp expression were determined by Western blot analysis using anti-HA. H, Quantitative analysis of the data from six independent experiments by densitometric measurements. For each sample, the band intensity of the processed, secreted Gbb-GFP was normalized by that of cell-associated Gbb-GFP to compensate for variations in transfection efficiency. Values shown represent the mean \pm SEM relative to the corresponding control (100\%). ${ }^{*} p<0.05 ;{ }^{* *} p<0.01$; ${ }^{* * *} p<0.001$.

A previous study has shown that Wsp is highly enriched postsynaptically at NMJs, and is also detected presynaptically at a lower level (Coyle et al., 2004). The same study has provided evidence for a presynaptic function of Wsp in restraining synaptic growth. However, no previous studies have addressed whether postsynaptic Wsp also contributes to synaptic growth regulation. In this study, our data demonstrate that Wsp also functions postsynaptically to regulate synaptic growth. First, postsynaptic knockdown of Wsp increases overall bouton number, satellite bouton formation, and NMJ length to levels similar to those observed in wsp-null mutants. Second, postsynaptic expression of
Wsp in wsp mutants reduces all synaptic growth parameters to wild-type levels.

\section{dCIP4/Wsp mode of action in the regulation of}

synaptic growth

Synaptic overgrowth caused by $d c i p 4, c d c 42$, wsp, or arp3 mutations is phenocopied by mutations in $d a d$, the inhibitory Smad gene in Drosophila, or overexpression of a constitutively active Tkv receptor (O'Connor-Giles et al., 2008), suggesting an inverse correlation between the activity of the postsynaptic $\mathrm{Cdc} 42-$ dCIP4-Wsp-Arp2/3 pathway and the levels of presynaptic BMP 
signaling and synaptic growth. Our genetic data support a model in which the postsynaptic Cdc42-dCIP4-Wsp-Arp2/3 pathway restrains presynaptic growth by attenuating retrograde BMP signaling. We show that presynaptic P-Mad levels at NMJs are significantly increased by RNAi-mediated knockdown of postsynaptic Cdc42, dCIP4, Wsp, Arp2, or Arp3. In addition, BMP signaling through the type II receptor Wit is necessary for synaptic overgrowth in larvae lacking postsynaptic dCIP4 or Wsp, suggesting that synaptic overgrowth in wsp is attributable to overactivation of retrograde BMP signaling. Finally, we have found that levels of extracellular Gbb at the NMJ are significantly increased by either dcip4 mutations or loss of postsynaptic Wsp.

How then does the postsynaptic dCIP4-Wsp-Arp $2 / 3$ pathway negatively regulate the retrograde Gbb signal? Previous studies have demonstrated that dCIP4 and Wsp function together in epithelial cells to promote E-cadherin endocytosis via the Arp2/3 complex and dynamin (Georgiou et al., 2008; Leibfried et al., 2008). Therefore, it is highly tempting to speculate that dCIP4 may antagonize the retrograde Gbb signal by promoting dynamin-dependent endocytosis of extracellular Gbb into muscles. However, our results suggest that this would not be the case. First, postsynaptic expression of a dominant-negative form of dynamin does not noticeably change extracellular Gbb levels at NMJs Second, the membrane-deforming activity of dCIP4, which has been implicated in endocytic vesicle formation (Fricke et al., 2009), is dispensable in the regulation of synaptic growth. Finally, depletion of dCIP4, Wsp, or Arp3 but not dynamin leads to an increase in the secretion of mature Gbb from cultured S2 cells, whereas dCIP4 overexpression has the opposite effect. Thus, our data support the notion that the dCIP4-Wsp-Arp2/3 pathway plays an inhibitory role in Gbb secretion.

The inhibitory role of Cdc42 and Wsp in Gbb secretion is consistent with a previous finding that overexpression of a constitutively active mutant of Cdc42 (Cdc42V12) and Wasp in mammalian HeLa cells inhibits $\mathrm{Cu}^{2+}$-dependent exocytosis of MNK (the Menkes disease protein) from the trans-Golgi network to the plasma membrane (Cobbold et al., 2002). In addition, overexpression of another constitutively active mutant of Cdc42 (Cdc42L61) has also been shown to inhibit glucose-stimulated insulin secretion from pancreatic $\beta$-cells (Nevins and Thurmond, 2003). In contrast, other studies have shown that overexpression of Cdc42L61 and N-Wasp enhances $\mathrm{Ca}^{2+}$-regulated exocytosis of growth hormone in PC12 neuroendocrine cells (Gasman et al., 2004). Thus, the effect of Cdc42 and Wasp overactivation on exocytosis appears to be different depending on the cell type or exocytic pathway.

Previous work has shown that retrograde BMP signaling through Wit is responsible for glutamate receptor-dependent synaptic homeostasis at the Drosophila NMJ (Haghighi et al., 2003). As glutamate receptors are $\mathrm{Ca}^{2+}$-permeable channels (Chang et al., 1994), it is possible that the Cdc42-dCIP4-WspArp $2 / 3$ pathway could be functionally connected to signal transduction molecules that respond to changes in glutamate receptor-dependent $\mathrm{Ca}^{2+}$ influx into postsynaptic muscles. Such candidate molecules include $\mathrm{Ca}^{2+} /$ calmodulin-dependent kinase II (CaMKII), which acts downstream of glutamate receptors to mediate retrograde signaling in muscles (Haghighi et al., 2003). Interestingly, it has been shown that CaMKII is activated by ganglioside-induced $\mathrm{Ca}^{2+}$ elevation and induces Cdc42mediated actin polymerization in mammalian cells (Chen et al., 2003). Therefore, it will be of great interest to investigate the regulation of the Cdc42-dCIP4-Wsp-Arp2/3 pathway by CaMKII and other related $\mathrm{Ca}^{2+}$ sensors in postsynaptic cells.

\section{References}

Aberle H, Haghighi AP, Fetter RD, McCabe BD, Magalhaes TR, Goodman CS (2002) wishful thinking encodes a BMP type II receptor that regulates synaptic growth in Drosophila. Neuron 33:545-558.

Bogdan S, Stephan R, Lobke C, Mertens A, Klambt C (2005) Abi activates WASP to promote sensory organ development. Nat Cell Biol 7:977-984.

Brand AH, Perrimon N (1993) Targeted gene expression as a means of altering cell fates and generating dominant phenotypes. Development 118:401-415

Budnik V, Koh YH, Guan B, Hartmann B, Hough C, Woods D, Gorczyca M (1996) Regulation of synapse structure and function by the Drosophila tumor suppressor gene dlg. Neuron 17:627-640.

Carroll RC, Beattie EC, Xia H, Luscher C, Altschuler Y, Nicoll RA, Malenka RC, von Zastrow M (1999) Dynamin-dependent endocytosis of ionotropic glutamate receptors. Proc Natl Acad Sci U S A 96:14112-14117.

Chang H, Ciani S, Kidokoro Y (1994) Ion permeation properties of the glutamate receptor channel in cultured embryonic Drosophila myotubes. J Physiol 476:1-16.

Chen N, Furuya S, Doi H, Hashimoto Y, Kudo Y, Higashi H (2003) Ganglioside/calmodulin kinase II signal inducing cdc42-mediated neuronal actin reorganization. Neuroscience 120:163-176.

Cobbold C, Ponnambalam S, Francis MJ, Monaco AP (2002) Novel membrane traffic steps regulate the exocytosis of the Menkes disease ATPase. Hum Mol Genet 11:2855-2866.

Coyle IP, Koh YH, Lee WC, Slind J, Fergestad T, Littleton JT, Ganetzky B (2004) Nervous wreck, an SH3 adaptor protein that interacts with Wsp, regulates synaptic growth in Drosophila. Neuron 41:521-534.

Doctor JS, Jackson PD, Rashka KE, Visalli M, Hoffmann FM (1992) Sequence, biochemical characterization, and developmental expression of a new member of the TGF-beta superfamily in Drosophila melanogaster. Dev Biol 151:491-505.

Estes PS, Jackson TC, Stimson DT, Sanyal S, Kelly LE, Ramaswami M (2003) Functional dissection of a eukaryotic dicistronic gene: transgenic stonedB, but not stonedA, restores normal synaptic properties to Drosophila stoned mutants. Genetics 165:185-196.

Fricke R, Gohl C, Dharmalingam E, Grevelhorster A, Zahedi B, Harden N, Kessels M, Qualmann B, Bogdan S (2009) Drosophila Cip4/Toca-1 integrates membrane trafficking and actin dynamics through WASP and SCAR/WAVE. Curr Biol 19:1429-1437.

Frost A, Perera R, Roux A, Spasov K, Destaing O, Egelman EH, De Camilli P, Unger VM (2008) Structural basis of membrane invagination by F-BAR domains. Cell 132:807-817.

Gasman S, Chasserot-Golaz S, Malacombe M, Way M, Bader MF (2004) Regulated exocytosis in neuroendocrine cells: a role for subplasmalemmal Cdc42/N-WASP-induced actin filaments. Mol Biol Cell 15:520-531.

Genova JL, Jong S, Camp JT, Fehon RG (2000) Functional analysis of Cdc42 in actin filament assembly, epithelial morphogenesis, and cell signaling during Drosophila development. Dev Biol 221:181-194.

Georgiou M, Marinari E, Burden J, Baum B (2008) Cdc42, Par6, and aPKC regulate Arp2/3-mediated endocytosis to control local adherens junction stability. Curr Biol 18:1631-1638.

Haghighi AP, McCabe BD, Fetter RD, Palmer JE, Hom S, Goodman CS (2003) Retrograde control of synaptic transmission by postsynaptic CaMKII at the Drosophila neuromuscular junction. Neuron 39:255-267.

Ho HY, Rohatgi R, Lebensohn AM, Le M, Li J, Gygi SP, Kirschner MW (2004) Toca-1 mediates Cdc42-dependent actin nucleation by activating the N-WASP-WIP complex. Cell 118:203-216.

Hudson AM, Cooley L (2002) A subset of dynamic actin rearrangements in Drosophila requires the Arp2/3 complex. J Cell Biol 156:677-687.

Jan LY, Jan YN (1982) Antibodies to horseradish peroxidase as specific neuronal markers in Drosophila and in grasshopper embryos. Proc Natl Acad Sci U S A 79:2700-2704.

Keshishian H, Kim YS (2004) Orchestrating development and function: retrograde BMP signaling in the Drosophila nervous system. Trends Neurosci 27:143-147.

Kim MD, Kamiyama D, Kolodziej P, Hing H, Chiba A (2003) Isolation of Rho GTPase effector pathways during axon development. Dev Biol 262:282-293.

Lahey T, Gorczyca M, Jia XX, Budnik V (1994) The Drosophila tumor suppressor gene dlg is required for normal synaptic bouton structure. Neuron 13:823-835.

Lee S, Harris KL, Whitington PM, Kolodziej PA (2000) short stop is allelic to 
kakapo, and encodes rod-like cytoskeletal-associated proteins required for axon extension. J Neurosci 20:1096-1108.

Lee S, Nahm M, Lee M, Kwon M, Kim E, Zadeh AD, Cao H, Kim HJ, Lee ZH, Oh SB, Yim J, Kolodziej PA (2007) The F-actin-microtubule crosslinker Shot is a platform for Krasavietz-mediated translational regulation of midline axon repulsion. Development 134:1767-1777.

Lee Y, Lee J, Bang S, Hyun S, Kang J, Hong ST, Bae E, Kaang BK, Kim J (2005) Pyrexia is a new thermal transient receptor potential channel endowing tolerance to high temperatures in Drosophila melanogaster. Nat Genet 37:305-310.

Leibfried A, Fricke R, Morgan MJ, Bogdan S, Bellaiche Y (2008) Drosophila Cip4 and WASp define a branch of the Cdc42-Par6-aPKC pathway regulating E-cadherin endocytosis. Curr Biol 18:1639-1648.

Lin DM, Goodman CS (1994) Ectopic and increased expression of Fasciclin II alters motoneuron growth cone guidance. Neuron 13:507-523.

Luo L, Liao YJ, Jan LY, Jan YN (1994) Distinct morphogenetic functions of similar small GTPases: Drosophila Dracl is involved in axonal outgrowth and myoblast fusion. Genes Dev 8:1787-1802.

Marie B, Sweeney ST, Poskanzer KE, Roos J, Kelly RB, Davis GW (2004) Dap160/intersectin scaffolds the periactive zone to achieve high-fidelity endocytosis and normal synaptic growth. Neuron 43:207-219.

Marques G, Zhang B (2006) Retrograde signaling that regulates synaptic development and function at the Drosophila neuromuscular junction. Int Rev Neurobiol 75:267-285.

Marques G, Bao H, Haerry TE, Shimell MJ, Duchek P, Zhang B, O'Connor MB (2002) The Drosophila BMP type II receptor Wishful Thinking regulates neuromuscular synapse morphology and function. Neuron 33: 529-543.

McCabe BD, Marques G, Haghighi AP, Fetter RD, Crotty ML, Haerry TE, Goodman CS, O'Connor MB (2003) The BMP homolog Gbb provides a retrograde signal that regulates synaptic growth at the Drosophila neuromuscular junction. Neuron 39:241-254.

Moline MM, Southern C, Bejsovec A (1999) Directionality of wingless protein transport influences epidermal patterning in the Drosophila embryo. Development 126:4375-4384.

Nevins AK, Thurmond DC (2003) Glucose regulates the cortical actin network through modulation of $\mathrm{Cdc} 42$ cycling to stimulate insulin secretion. Am J Physiol Cell Physiol 285:C698-C710.

O'Connor-Giles KM, Ho LL, Ganetzky B (2008) Nervous wreck interacts with thickveins and the endocytic machinery to attenuate retrograde BMP signaling during synaptic growth. Neuron 58:507-518.
Osterwalder T, Yoon KS, White BH, Keshishian H (2001) A conditional tissue-specific transgene expression system using inducible GAL4. Proc Natl Acad Sci U S A 98:12596-12601.

Persson U, Izumi H, Souchelnytskyi S, Itoh S, Grimsby S, Engstrom U, Heldin CH, Funa K, ten Dijke P (1998) The L45 loop in type I receptors for TGF-beta family members is a critical determinant in specifying Smad isoform activation. FEBS Lett 434:83-87.

Ramachandran P, Barria R, Ashley J, Budnik V (2009) A critical step for postsynaptic F-actin organization: regulation of Baz/Par-3 localization by aPKC and PTEN. Dev Neurobiol 69:583-602.

Rawson JM, Lee M, Kennedy EL, Selleck SB (2003) Drosophila neuromuscular synapse assembly and function require the TGF-beta type I receptor saxophone and the transcription factor Mad. J Neurobiol 55:134-150.

Regehr WG, Carey MR, Best AR (2009) Activity-dependent regulation of synapses by retrograde messengers. Neuron 63:154-170.

Robertson HM, Preston CR, Phillis RW, Johnson-Schlitz DM, Benz WK, Engels WR (1988) A stable genomic source of P element transposase in Drosophila melanogaster. Genetics 118:461-470.

Rodal AA, Motola-Barnes RN, Littleton JT (2008) Nervous wreck and Cdc42 cooperate to regulate endocytic actin assembly during synaptic growth. J Neurosci 28:8316-8325.

Stewart BA, Atwood HL, Renger JJ, Wang J, Wu CF (1994) Improved stability of Drosophila larval neuromuscular preparations in haemolymphlike physiological solutions. J Comp Physiol A Neuroethol Sens Neural Behav Physiol 175:179-191.

Strigini M, Cohen SM (2000) Wingless gradient formation in the Drosophila wing. Curr Biol 10:293-300.

Sweeney ST, Davis GW (2002) Unrestricted synaptic growth in spinster-a late endosomal protein implicated in TGF-beta-mediated synaptic growth regulation. Neuron 36:403-416.

Tao HW, Poo M (2001) Retrograde signaling at central synapses. Proc Natl Acad Sci U S A 98:11009-11015.

Tian L, Nelson DL, Stewart DM (2000) Cdc42-interacting protein 4 mediates binding of the Wiskott-Aldrich syndrome protein to microtubules. J Biol Chem 275:7854-7861.

Wang X, Shaw WR, Tsang HT, Reid E, O'Kane CJ (2007) Drosophila spichthyin inhibits BMP signaling and regulates synaptic growth and axonal microtubules. Nat Neurosci 10:177-185.

Wodarz A, Hinz U, Engelbert M, Knust E (1995) Expression of crumbs confers apical character on plasma membrane domains of ectodermal epithelia of Drosophila. Cell 82:67-76. 